\title{
On-chip zero-index metamaterials
}

\section{Citation}

Li, Yang, Shota Kita, Philip Muñoz, Orad Reshef, Daryl I. Vulis, Mei Yin, Marko Loncar, and Eric Mazur. 2015. “On-Chip Zero-Index Metamaterials." Nature Photon 9 (11) (October 19): 738-742. doi:10.1038/nphoton.2015.198. http://dx.doi.org/10.1038/nphoton.2015.198.

\section{Published Version}

doi:10.1038/nphoton.2015.198

\section{Permanent link}

http://nrs.harvard.edu/urn-3:HUL.InstRepos:25623047

\section{Terms of Use}

This article was downloaded from Harvard University's DASH repository, and is made available under the terms and conditions applicable to Other Posted Material, as set forth at http:// nrs.harvard.edu/urn-3:HUL.InstRepos:dash.current.terms-of-use\#LAA

\section{Share Your Story}

The Harvard community has made this article openly available.

Please share how this access benefits you. Submit a story.

Accessibility 


\title{
Title: On-chip zero-index metamaterials
}

\author{
Authors: Yang $\mathrm{Li}^{1} \uparrow$, Shota $\mathrm{Kita}^{1} \uparrow$, Philip Muñoz ${ }^{1}$, Orad Reshef ${ }^{1}$, Daryl I. Vulis ${ }^{1}$, Mei Yin $^{1,2}$, \\ Marko Lončar ${ }^{1 *}$, and Eric Mazur ${ }^{1,3 *}$
}

Summary: Metamaterials with a refractive index of zero exhibit physical properties such as infinite phase velocity and wavelength. However, there is no way to implement these materials on a photonic chip, restricting the investigation and application of zero-index phenomena to simple shapes and small scales. We designed and fabricated an on-chip integrated metamaterial with a refractive index of zero in the optical regime. Light refracts perpendicular to the facets of a prism made of this metamaterial, directly demonstrating that the index of refraction is zero. The metamaterial consists of low-aspect-ratio silicon pillar arrays embedded in a polymer matrix and clad by gold films. This structure can be fabricated using standard planar processes over a large area in arbitrary shapes and can efficiently couple to photonic integrated circuits and other optical elements. This novel on-chip metamaterial platform opens the door to exploring the physics of zero-index and its applications in integrated optics.

\footnotetext{
${ }^{1}$ School of Engineering and Applied Sciences, Harvard University, 9 Oxford Street, Cambridge, Massachusetts 02138, USA.

${ }^{2}$ State Key Laboratory of Advanced Optical Communication Systems and Networks, School of Electronics

Engineering and Computer Science, Peking University, 5 Yiheyuan Road, Beijing 100871, China.

${ }^{3}$ Department of Physics, Harvard University, 9 Oxford Street, Cambridge, Massachusetts 02138, USA.

$\dagger$ These authors contributed equally to this work.

*e-mail: loncar@seas.harvard.edu; mazur@seas.harvard.edu
} 


\section{Introduction}

Metamaterials — composite materials whose electromagnetic properties are engineered by structuring their constituents ${ }^{1,2}$ - make it possible to achieve a refractive index equal to zero ${ }^{3}$. When the index of refraction is zero, the phase velocity is infinite. In this extreme limit, the wavelength inside the material is infinite and the phase is uniform throughout, regardless of its overall size and shape. All fields within the material oscillate in unison, achieving electrostatic behavior at optical frequencies ${ }^{4}$. This regime allows access to a wealth of exciting physical phenomena and potential applications including super-coupling, cloaking, and new approaches for phase matching in nonlinear optics ${ }^{3,5-9}$.

Experimental demonstrations of zero-index metamaterials typically involve metals operating around their plasma frequencies ${ }^{10-12}$ or metallic resonators ${ }^{13-16}$, resulting in high loss and impedance mismatch. Recently, zero index was demonstrated by tuning the Mie resonances in a purely dielectric photonic crystal structure ${ }^{17,18}$. This approach offers several important advantages. First, dielectric metamaterials avoid the losses associated with metals, especially in the optical regime. Second, tuning the Mie resonances allows for the simultaneous control of the electric and magnetic response ${ }^{19,20}$. In particular, when the effective relative permittivity $\varepsilon_{\mathrm{r}}^{\text {eff }}$ and permeability $\mu_{\mathrm{r}}^{\mathrm{eff}}$ approach zero simultaneously, the impedance $\eta_{\mathrm{eff}}=\sqrt{\mu_{\mathrm{r}}^{\mathrm{eff}} / \varepsilon_{\mathrm{r}}^{\mathrm{eff}}}$ can be matched to that of other materials.

For integrated photonic applications, zero-index metamaterials require light to be confined on-chip and thus need to be realized in an in-plane geometry (that is, with the light propagating parallel to the substrate). This geometry would permit integrating these metamaterials with other optical elements, including waveguides, resonators, and interferometers. To date, zero-index 
metamaterials have been demonstrated only in an out-of-plane geometry, with functional layers of the metamaterials stacked on a substrate and where light propagates normal to the sample surface $^{12,16,18}$. This geometry is not only impractical for integrated-photonics applications, but is also limited to short interaction lengths, and cannot be fabricated in arbitrary shapes.

In this paper, we present an on-chip, in-plane metamaterial with zero index in the telecom wavelength range. Our design consists of a square array of low-aspect-ratio silicon pillars on a silicon-on-insulator (SOI) substrate. The array is embedded in an SU-8 slab waveguide, and clad above and below by gold films. We fabricate this metamaterial using conventional nanofabrication processes (Fig. 1a). First, we define an array of pillars in a 512-nm-thick silicon device layer using electron-beam lithography followed by inductively coupled plasma reactive ion etching (step I in Fig. 1a). Next, we use electron-beam evaporation to deposit a 50-nm-thick gold film (step II). We then embed the pillars within a 595-nm-thick SU-8 photoresist layer (step III). Finally, a second gold film is deposited to complete the fabrication process (step IV). We can produce arbitrarily shaped pillar arrays using such conventional planar fabrication techniques in order to realize a variety of metamaterial devices.

We demonstrate zero index by measuring the refraction through a prism made of this metamaterial (Fig. 1b) with a 1.5- $\mu$ m-thick SU-8 matrix (see last paragraph of the section: design) (Supplementary Information Section 1). A silicon waveguide oriented perpendicular to one of the prism facets guides the incident beam (Fig. 1b). Light that enters the prism is refracted at the output facet into a semi-circular slab of SU-8, which we use to determine the angle of refraction (Fig. 1b, c). The measured angles of refraction are in excellent agreement with simulations and 
unambiguously demonstrate that the metamaterial prism has zero index at a wavelength of 1570 nm.

\section{Design}

Previous designs for dielectric zero-index metamaterials are based on infinitely long silicon pillars ${ }^{17,18}$. These designs are incompatible with integrated silicon photonic platforms, which are typically based on silicon device layers with thickness on the order of the wavelength. One can replicate the behavior of infinitely long pillars by placing short silicon pillars between parallel conductors $^{21}$. This approach reduces the out-of-plane radiation loss and enforces the fundamental TM mode by ensuring that the electric field between the parallel conductors is along the pillar axis (Supplementary Information Section 2). So, it preserves the impedance-matched zero index using a structure of finite height, and allows the metamaterial to be integrated with conventional nanophotonic components (Fig. 1). We adjust the pitch and radius of the silicon pillar array to obtain zero index with low propagation loss at a wavelength of $1590 \mathrm{~nm}$, near the middle of the 1480 to 1680 -nm tuning range of our laser (Supplementary Information Section 3). Propagation loss in this metamaterial is dominated by the conduction loss originating from the gold films (Supplementary Information Section 4).

To characterize the optical properties of the designed metamaterial, we calculate its band structure in the plane of the array (Fig. 2a). As Fig. 2a shows, two linear dispersion bands intersect at the $\Gamma$ point. These bands form a Dirac-like cone (blue, Fig. 2b) intersecting a quadratic dispersion band (red) at the Dirac point at the center of the Brillouin zone ${ }^{17}$. The two lineardispersion bands correspond to electric monopole and transverse magnetic dipole modes near the Dirac point (Fig. 2c). At the Dirac point, the wavenumber approaches zero, indicating that the effective index of the metamaterial is also zero. Furthermore, the nearly circular isofrequency 
contours in a 55-nm bandwidth around the Dirac point (Fig. 2d) suggest that the metamaterial is essentially isotropic, producing a refractive index of zero in almost all propagation directions. The effective wavelength $\lambda_{\text {eff }}$ approaches infinity in the vicinity of the $\Gamma$ point, satisfying the homogenization criterion ${ }^{17,18,22}$ and allowing us to treat the metamaterial as a homogeneous bulk medium with effective constitutive parameters in the vicinity of the Dirac point (Supplementary Information Section 5). We can retrieve the effective relative permittivity $\varepsilon_{\mathrm{r}}^{\text {eff }}$ and permeability $\mu_{\mathrm{r}}^{\mathrm{eff}}$ of this metamaterial from the simulated reflection and transmission coefficients (Fig. 2e) ${ }^{23}$. The computed $\varepsilon_{\mathrm{r}}^{\text {eff }}$ and $\mu_{\mathrm{r}}^{\text {eff }}$ cross zero simultaneously and linearly at the design wavelength of $1590 \mathrm{~nm}$, with an effective impedance of 1.47. This simultaneous and linear electric and magnetic response is a unique characteristic of zero-index metamaterials corresponding to Dirac cones, and is essential to achieve a finite impedance ${ }^{17}$. The $\varepsilon_{\mathrm{r}}^{\text {eff }}$ and $\mu_{\mathrm{r}}^{\text {eff }}$ are only linear in the vicinity of the Dirac-point wavelength ${ }^{17}$. All of these results indicate that this metamaterial possesses a relatively isotropic zero effective index with good impedance matching to free space and to standard optical waveguides.

Our simulations show that a Dirac cone can be obtained even for significantly larger SU-8 layer thicknesses (Supplementary Information Section 6), providing more flexibility in the fabrication process. Because it is difficult to spin on a very thin SU-8 layer between the silicon pillars right next to a large tapered waveguide, we chose to use a $1.5-\mu \mathrm{m}$-thick, rather than a 595nm-thick SU-8 layer in the fabrication of our prisms (Fig. 1b, c).

\section{Experimental results}

To experimentally measure the refractive index of the metamaterial, we illuminate the metamaterial prism and measure the angle of the refracted beam (Fig. 1b, c). The prism is 
illuminated with TM-polarized light delivered via the tapered silicon waveguide. The light propagates through the prism with an effective index $n_{1}^{\text {eff }}$, and reaches the interface between the prism and a SU-8 slab waveguide at an angle of incidence of $45^{\circ}$. The refracted beam then propagates as a guided mode of the SU-8 slab waveguide with known effective index $n_{2}^{\text {eff }}$, until it is scattered at the semi-circular edge of SU-8 slab waveguide (Supplementary Information Section 7). The scattered light is imaged from above using an infrared camera to determine the refraction angle $\alpha$. This refraction angle is used to determine $n_{1}^{\text {eff }}$ using Snell's law: $n_{2}^{\text {eff }} / n_{1}^{\text {eff }}=\sin 45^{\circ} / \sin \alpha$.

Fig. 3a shows the experimentally observed refraction in the prism and the SU-8 slab waveguide at $\lambda=1570 \mathrm{~nm}$, where the metamaterial shows zero index (Supplementary Information Section 8). Because of fabrication imperfections, this wavelength is slightly different from the design wavelength of $1590 \mathrm{~nm}$. The yellow arrow in Fig. 3a shows the refracted beam, which propagates perpendicular to the interface between the prism and SU-8 slab waveguide, corresponding to a prism with a refractive index of zero. The prism also generates several side beams at $\lambda=1570 \mathrm{~nm}$ due to additional modes in the band structure (Fig. 2a) (Supplementary Information Section 9).

Fig. $3 \mathrm{~b}$ shows the corresponding numerical calculation of the out-of-plane electromagnetic field distribution in the prism and the SU-8 slab waveguide region at $\lambda=1570 \mathrm{~nm}$. As in the observed results, the refracted beam propagates perpendicular to the interface between the prism and SU-8 slab waveguide, with several side beams appearing on either side. As shown in the 
magnified view on the right in Fig. 3b, there is no spatial phase variation within the prism, as the effective wavelength is infinite inside the metamaterial.

We probe the dispersion of the metamaterial index by measuring the angle of refraction while varying the input wavelength from 1480 to $1680 \mathrm{~nm}$. The left panel of Fig. 3c shows the intensity measured along the curved output edge of the SU-8 slab waveguide as a function of wavelength and refraction angle. The right panel of Fig. 3c shows the corresponding simulated far-field pattern (Supplementary Information Section 10). We observe excellent agreement between the measured and simulated far-field patterns, with both patterns showing the refracted beam in the center, near $0^{\circ}$. The shifting of the refracted beam indicates that the refractive index of the prism continuously changes from a positive value at shorter wavelengths to a negative value at longer wavelengths.

Using Snell's law, we extract the index of refraction of the metamaterial from the position of the refracted beam in the wavelength range of 1480-1680 nm (Supplementary Information Section 11). As shown in Fig. 3d, the measured index varies from $0.15 \pm 0.04$ at $1480 \mathrm{~nm}$ to $-0.57 \pm 0.04$ at $1680 \mathrm{~nm}$, exhibiting linear dispersion near the zero crossing at $1570 \mathrm{~nm}$. The error bars represent uncertainties in the measured index due to finite image resolution and fitting uncertainty (Supplementary Information Section 11). Using a similar extraction method, we calculate the index of refraction from the simulated far-field patterns, showing excellent agreement with measured values.

To determine whether our measured zero index is caused by a Dirac cone or by a small band gap, we measured two prisms with both smaller and larger pillar radii. Theoretically, a photonic Dirac cone has no band gap at the Dirac-point wavelength. Due to fabrication imperfections, however, a tiny band gap may open near the targeted Dirac-point wavelength of the 
fabricated zero-index metamaterial. In that case, the real part of the index has a constant zero value rather than linear dispersion. Given our error bars (Fig. 3d), the band gap is at most $50 \mathrm{~nm}$ wide. Index measurements of prisms with smaller or larger radii show that the band gaps of these metamaterials are blue-shifted or red-shifted, respectively. In addition, the bandgaps of those with larger radii become significantly wider. This behavior confirms that the experimentally measured zero index in Fig. 3 corresponds to a Dirac cone at the $\Gamma$ point ${ }^{17}$ (Supplementary Information Section 12).

As a control, we also measured the refractive index in the absence of the metamaterial prism. In this control experiment, light from the input silicon waveguide propagates through free space before refracting into the SU-8 slab waveguide. The measured index of the void is $0.94 \pm 0.04$ in the wavelength range $1480-1680 \mathrm{~nm}$, in good agreement with the index of air (Supplementary Information Section 13). Therefore, the measured index shown in Fig. 3 corresponds to the effective index of the metamaterial prism, rather than an artifact of the measurement setup.

\section{Conclusion}

We experimentally demonstrate the first on-chip integrated zero-index metamaterial in the optical regime and directly measure the effective index of a prism consisting of this material. The metamaterial is effectively two-dimensional (2D), as energy propagates in the plane of the array. This in-plane structure can efficiently couple to silicon waveguides to interface with standard integrated photonic components. Using standard planar processes, the metamaterial can be fabricated over a large area with high fidelity and in arbitrary shapes. This design enables direct implementation of zero-index phenomena on a chip, including super-couplers, surface-emitting lasers, and new approaches for phase matching in nonlinear optics ${ }^{3,5,8,9,24}$. It can also serve as an 
on-chip lab to explore fundamental quantum science such as photon entanglement and enhancement of spontaneous emission ${ }^{4,18}$.

\section{References}

1. Soukoulis, C. M. \& Wegener, M. Past achievements and future challenges in the development of three-dimensional photonic metamaterials. Nat. Photonics 5, 523-530 (2011).

2. Engheta, N. \& Ziolkowski, R. W. Metamaterials: Physics and Engineering Explorations (Wiley, New York, 2006).

3. Engheta, N. Pursuing near-zero response. Science 340, 286-287 (2013).

4. Mahmoud, A. M. \& Engheta, N. Wave-matter interactions in epsilon-and-mu-near-zero structures. Nat. Commun. 5, 5638 (2014).

5. Silveirinha, M. \& Engheta, N. Tunneling of electromagnetic energy through subwavelength channels and bends using $\varepsilon$-near-zero materials. Phys. Rev. Lett. 97, 157403 (2006).

6. Hao, J. M., Yan, W. \& Qiu, M. Super-reflection and cloaking based on zero index metamaterial. Appl. Phys. Lett. 96, 101109 (2010).

7. Nguyen, V. C., Chen, L. \& Klaus, H. Total transmission and total reflection by zero index metamaterials with defects. Phys. Rev. Lett. 105, 233908 (2010).

8. Rose, A. \& Smith, D. R. Overcoming phase mismatch in nonlinear metamaterials. Opt. Mater. Express 1, 1232-1243 (2011).

9. Suchowski, H. et al. Phase mismatch-free nonlinear propagation in optical zero-index materials. Science 342, 1223-1226 (2013). 
10. Pendry, J. B., Holden, A. J., Stewart, W. J. \& Youngs, I. Extremely low frequency plasmons in metallic mesostructures. Phys. Rev. Lett. 76, 4773-4776 (1996).

11. Boltasseva, A. \& Atwater, H. A. Low-loss plasmonic metamaterials. Science 331, 290-291 (2011).

12. Maas, R., Parsons, J., Engheta, N. \& Polman, A. Experimental realization of an epsilon-nearzero metamaterial at visible wavelengths. Nat. Photonics 7, 907-912 (2013).

13. Pendry, J. B., Holden, A. J., Robbins, D. J. \& Stewart, W. J. Magnetism from conductors and enhanced nonlinear phenomena. IEEE Trans. Microw. Theory Tech. 47, 2075-2084 (1999).

14. Shelby, R. A., Smith, D. R. \& Schultz, S. Experimental verification of a negative index of refraction. Science 292, 77-79 (2001).

15. Liu, R. et al. Experimental demonstration of electromagnetic tunneling through an epsilonnear-zero metamaterial at microwave frequencies. Phys. Rev. Lett. 100, 023903 (2008).

16. Valentine, J. et al. Three-dimensional optical metamaterial with a negative refractive index. Nature 455, 376-379 (2008).

17. Huang, X. Q., Lai, Y., Hang, Z. H., Zheng, H. H. \& Chan, C. T. Dirac cones induced by accidental degeneracy in photonic crystals and zero-refractive-index materials. Nat. Mater. 10, $582-586(2011)$

18. Moitra, P. et al. Realization of an all-dielectric zero-index optical metamaterial. Nat. Photonics 7, 791-795 (2013).

19. Staude, I. et al. Tailoring directional scattering through magnetic and electric resonances in subwavelength silicon nanodisks. ACS Nano 7, 7824-7832 (2013). 
20. Evlyukhin, A. B. et al. Optical spectroscopy of single Si nanocylinders with magnetic and electric resonances. Sci. Rep. 4, 4126 (2014).

21. Balanis, C. A. Engineering Electromagnetics (Wiley, Hoboken, 1989).

22. Simovski, C. R. On electromagnetic characterization and homogenization of nanostructured metamaterials. J. Opt. 13, 013001 (2011).

23. Smith, D. R., Vier, D. C., Koschny, T. \& Soukoulis, C. M. Electromagnetic parameter retrieval from inhomogeneous metamaterials. Phys. Rev. E 71, 036617 (2005).

24. Chua, S.-L., Lu, L., Bravo-Abad, J., Joannopoulos, J. D. \& Soljačić, M. Larger-area singlemode photonic crystal surface-emitting lasers enabled by an accidental Dirac point. Opt. Lett. 39, 2072-2075 (2014).

25. Wu, Y., Li, J., Zhang, Z. Q. \& Chan, C. T. Effective medium theory for magnetodielectric composites: Beyond the long-wavelength limit. Phys. Rev. B 74, 085111 (2006).

26. Hosseini, A. et al. On the role of evanescent modes and group index tapering in slow light photonic crystal waveguide coupling efficiency. Appl. Phys. Lett. 98, 031107, (2011).

27. Kita, S. et al. in CLEO: 2015. FM3C.2 (Optical Society of America).

28. Englund, D. et al. Controlling cavity reflectivity with a single quantum dot. Nature 450, 857861 (2007).

29. Hsu, C. W. et al. Observation of trapped light within the radiation continuum. Nature 499, 188-191 (2013).

30. Balanis, C. A. Antenna Theory: Analysis and Design, 3rd Edition (Wiley, New Jersey, 2005). 
31. Piggott, A. Y. et al. Inverse design and demonstration of a compact and broadband on-chip wavelength demultiplexer. Nat. Photonics 9, 374-377 (2015).

32. Shen, B., Wang, P., Polson, R. \& Menon, R. An integrated-nanophotonics polarization Beam splitter with $2.4 \times 2.4 \mu \mathrm{m}^{2}$ footprint. Nat. Photonics 9, 378-382 (2015).

33. Shore, R. A. \& Yaghjian, A. D. Traveling waves on two- and three-dimensional periodic arrays of lossless scatterers. Radio Sci. 42, RS6S21 (2007).

34. Simovski, C. R. \& Tretyakov, S. A. Local constitutive parameters of metamaterials from an effective-medium perspective. Phys. Rev. B 75, 195111 (2007).

35. Alù, A., Yaghjian, A. D., Shore, R. A. \& Silveirinha, M. G. Causality relations in the homogenization of metamaterials. Phys. Rev. B 84, 054305 (2011).

36. Yariv, A. Optical Electronics, 4th Edition (Oxford University Press, Oxford, 1990).

37. Hutley, M. Diffraction Gratings (Techniques of Physics) (Academic Press, London, 1982).

38. (ISO), G. I. O. f. S. (Geneva, 1993).

Supplementary Information is available in the online version of the paper.

\section{Acknowledgments}

The authors would like to acknowledge X. Q. Huang, Y. Wu, C. Simovski, C. C. Evans, Y. M. Yang for discussion, N. Liu for assistance with FDTD simulations, Q. M. Quan for assistance with the measurements, M. Kats for providing the measured complex index of gold, and 
K. Phillips for guidance on the preparation of the figures. The research described in this paper was supported by the National Science Foundation under contract DMR-1360889, the Air Force Office of Scientific Research under contract FA9550-14-1-0389, the Natural Sciences and Engineering Research Council of Canada, and the Harvard Quantum Optics Center.

\section{Author contributions}

Several people contributed to the work described in this paper. Y. Li conceived the basic idea for this work. Y. Li, P. Muñoz, and D. Vulis carried out the FDTD simulations. S. Kita, O. Reshef, and M. Yin carried out the FEM simulations. O. Reshef designed the optical waveguides. S. Kita performed the fabrication. S. Kita, O. Reshef, and D. Vulis carried out the measurements. P. Muñoz analyzed the experimental results. M. Lončar and E. Mazur supervised the research and the development of the manuscript. Y. Li wrote the first draft of the manuscript; all authors subsequently took part in the revision process and approved the final copy of the manuscript.

\section{Author information}

Reprints and permissions information is available at www.nature.com/reprints. The authors declare no competing financial interests. Readers are welcome to comment on the online version of the paper. Correspondence and requests for materials should be addressed to E.M. (mazur@seas.harvard) and M.L. (1oncar@seas.harvard.edu). 


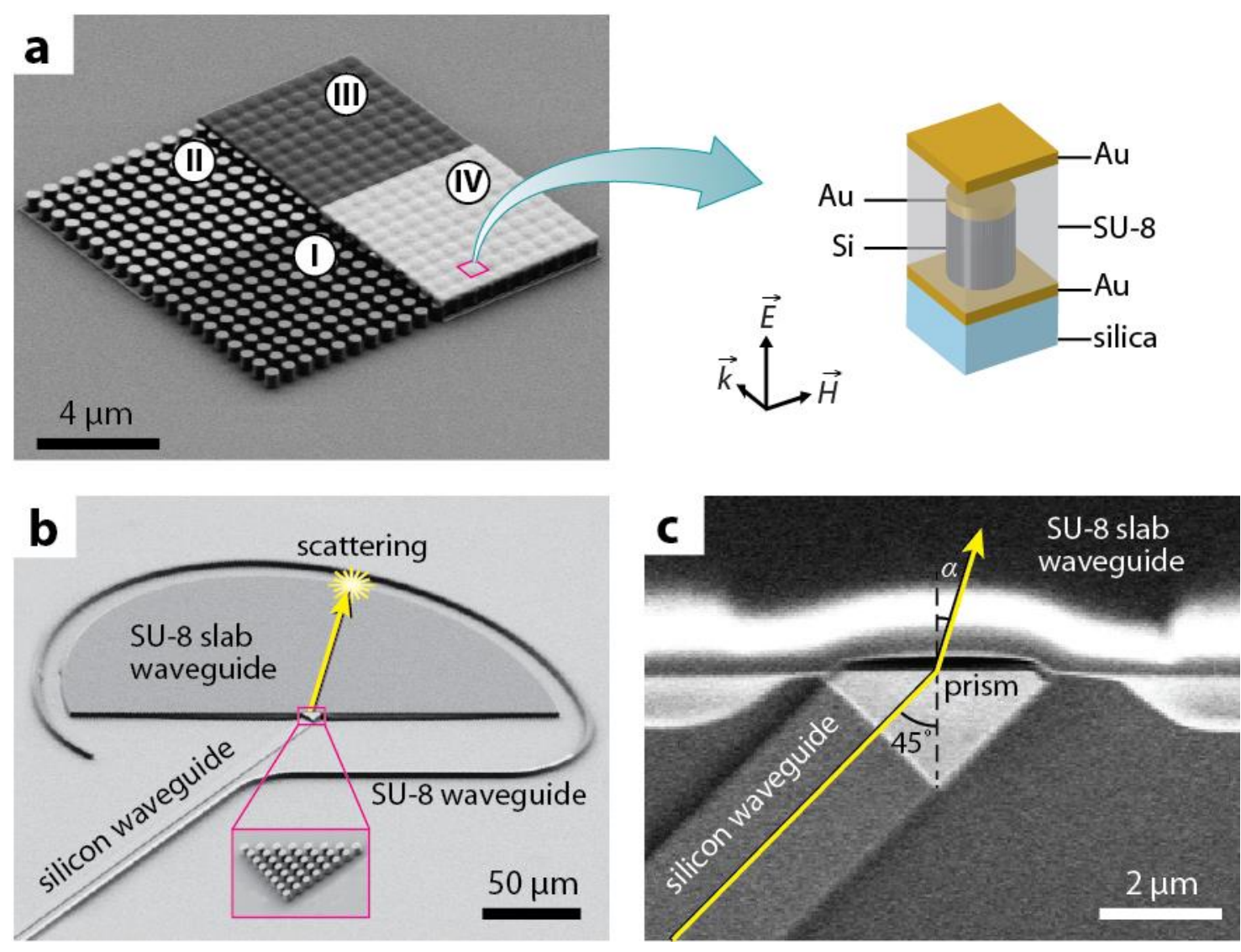

Figure 1. Metamaterial design and structure fabricated to demonstrate in-plane zero index.

a, SEM image of the metamaterial in different fabrication stages: I. Silicon pillars etched from SOI substrate; II. With bottom gold film; III. Embedded in SU-8 matrix; IV. Completed structure with top gold film. Inset: three-dimensional schematic of one unit-cell of the metamaterial. The pitch and radius of the silicon pillars are $690 \mathrm{~nm}$ and $211 \mathrm{~nm}$, respectively. b, A silicon waveguide carries the incident beam toward the metamaterial prism, where the beam is refracted into the SU8 slab waveguide. Inset shows the prism, which is a right triangular array of pillars measuring 8 unit cells across, without gold and SU-8 layers. c, Prism region showing the incident and refracted beams. The angle of refraction $\alpha$ is determined by measuring the position of the refracted beam at the curved output edge of SU-8 slab waveguide (yellow scattering spot in b). 

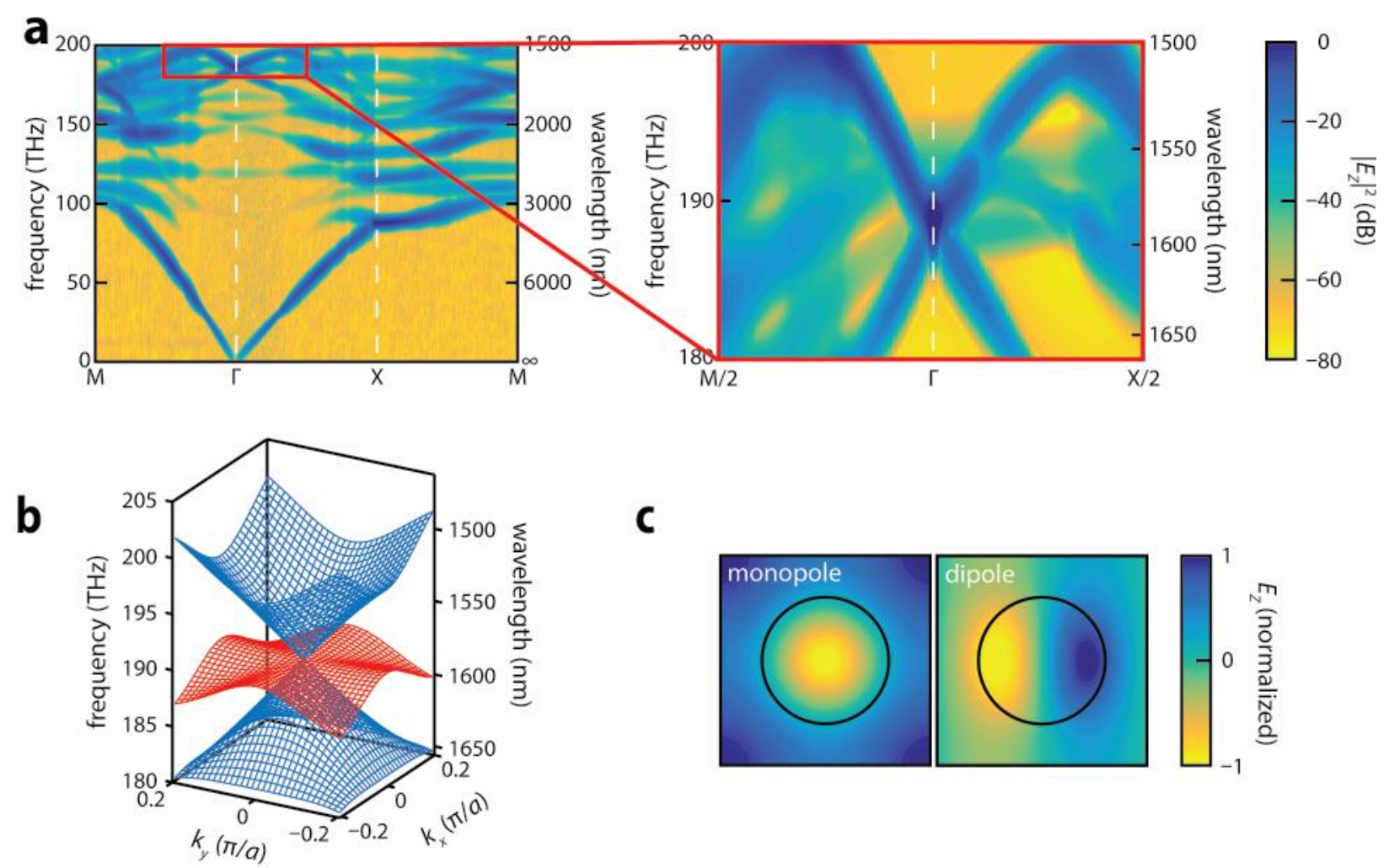

C
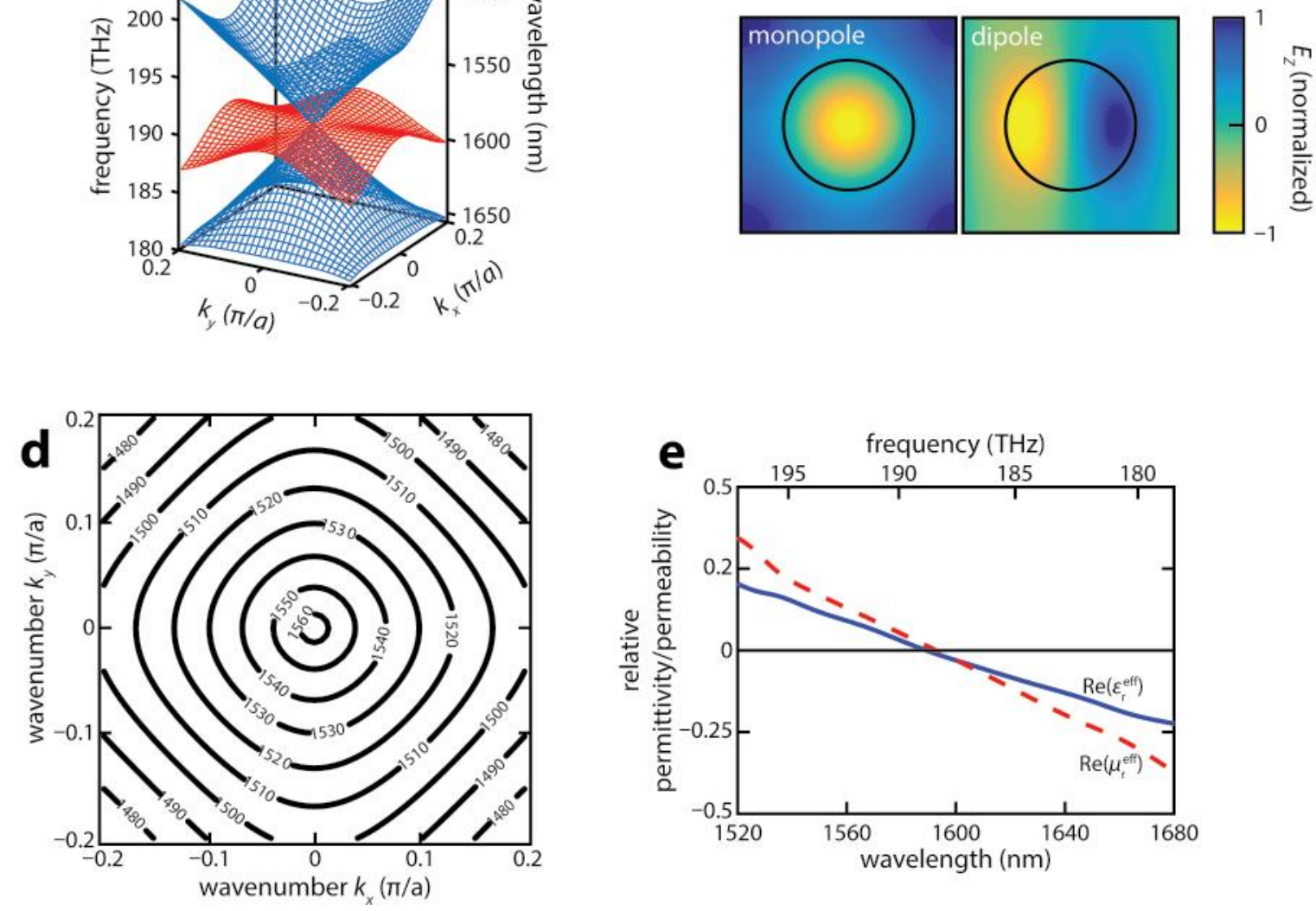

Figure 2. Optical properties of the zero-index metamaterial. a, Photonic band structure of the zero-index metamaterial (Fig. 1a) for TM modes. Two linear dispersion bands intersect at the $\Gamma$ point at $\lambda=1590 \mathrm{~nm}$. b, 3D dispersion surfaces. The linear bands (blue) form a Dirac-like cone. The quadratic band (red) is a quasi-longitudinal mode ${ }^{17}$. We show only the three modes that form the cone to emphasize the Dirac-cone dispersion clearly. c, Electric fields at the Dirac point over 
a unit-cell cross-section in the plane of the array, corresponding to an electric monopole mode and a transverse magnetic dipole mode. The black circles indicate the boundary of the silicon pillar. $\mathbf{d}$, Isofrequency contours of the zero-index metamaterial. The nearly circular contours indicate that this metamaterial is almost isotropic near the $\Gamma$ point. e, Effective relative permittivity and permeability of the metamaterial retrieved from simulated reflection and transmission coefficients.

Both parameters cross zero simultaneously resulting in finite impedance. 

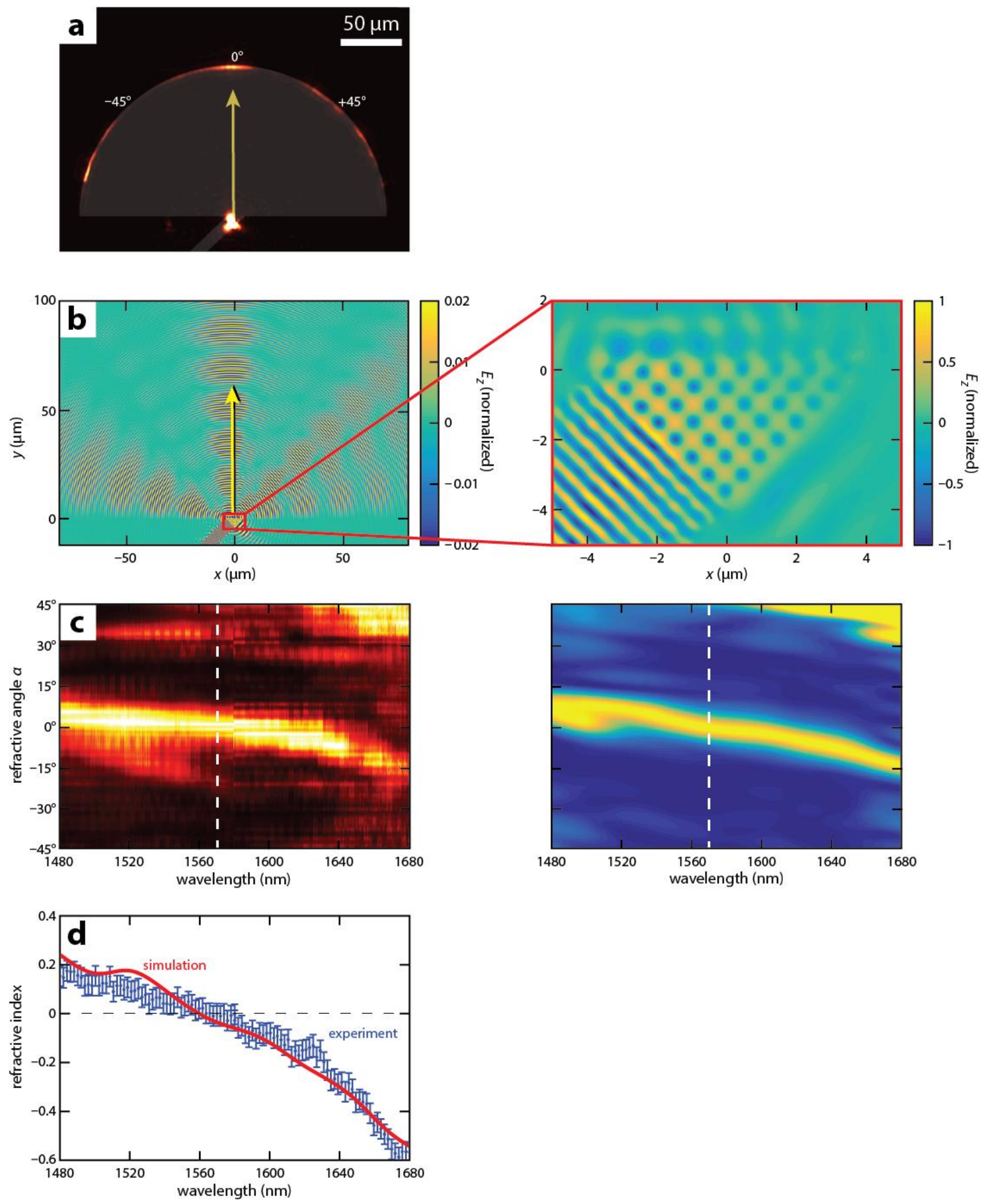

Figure 3. Simulation and experimental results. a, Near-infrared microscope image of the prism (Fig. 1b, c) at $1570 \mathrm{~nm}$, showing the refracted beam, which propagates normal to the interface 
between prism and SU-8 slab waveguide. The gray area corresponds to the silicon waveguide and SU-8 slab waveguide. $\mathbf{b}$, Left: simulated out-of-plane electric field $(\lambda=1570 \mathrm{~nm})$ in the prism and SU-8 slab waveguide region. The refracted beam is visible at the curved output edge of SU-8 slab waveguide at $0^{\circ}$. Right: magnified view of electric field distribution in the prism, illustrating nearly constant spatial phase distribution. c, Measured (left) and simulated (right) far-field patterns. The white dashed line indicates the wavelength, $1570 \mathrm{~nm}$, at which the refracted beam crosses $0^{\circ}$. The image is normalized at each wavelength. $\mathbf{d}$, Measured and simulated effective index of the zero-index metamaterial. The blue dots indicate measured refractive index, with error bars representing uncertainties in the measurement.

\section{Methods}

\section{Simulations}

We measure complex indices of gold, silicon, and silica via spectroscopic ellipsometry for use in our numerical simulations.

We calculate the complex reflection and transmission coefficients, electromagnetic field profiles, and far-field patterns using 3D finite-difference time-domain (FDTD) simulations. We extract the reflected and transmitted electric fields at two points prior to the source and after the metamaterial, respectively, to obtain the complex reflection and transmission coefficients (Fig. S26 of Supplementary Information). We collect the electric fields from a near-field simulation to obtain the far-field pattern using far-field projection.

We compute the band structures, dispersion surfaces, and isofrequency contours using $3 \mathrm{D}$ finite-element-method (FEM) simulations. We obtain these results by first calculating all the 
modes in a unit cell of our metamaterial with Floquet periodic boundary conditions in $x$ and $y$ directions and perfectly matched layers at the boundaries in the $z$ direction. We select the TMpolarized modes by evaluating the energy ratio of electric fields in $x, y$, and $z$ directions. We filter out the modes with low quality factors $(<10)$ or low core confinements in the metamaterial region.

\section{Fabrication}

We fabricate the zero-index metamaterial on a silicon-on-insulator (SOI) wafer using standard lithographic techniques (Fig. 1). Beginning with a 512-nm-thick silicon layer, the silicon pillars and coupling waveguides are patterned into negative-tone resist (XR-1541 6\%, Dow Corning) using electron-beam lithography (EBL) and are subsequently structured using inductively coupled plasma reactive ion etching (ICP-RIE). A layer of positive-tone resist (PMMA 495 C6, MicroChem) is spin-coated and patterned using EBL, designating the location of the bottom gold film. A 5-nm-thick titanium adhesion layer and a 95-nm-thick gold layer are deposited using e-beam evaporation and are subsequently lifted off by acetone boiling and very weak sonication to serve as the bottom gold film. Following this step, we spin-coat a $1.5-\mu \mathrm{m}$-thick SU8 layer and cure it using EBL, forming the polymer matrix around the silicon pillars to support the top gold film. The steps to structure and deposit the top gold film are the same as those for the bottom gold film.

To make the four different parts showing each fabrication step (Fig. 1a), we used positive resist openings for selective gold deposition on the individual parts, following the fabrication procedure outlined above. 


\title{
Supplementary Information:
}

Materials and Methods

Figures: S1-S26

Videos: video_S1_Ez_1530nm, video_S2_Ez_1590nm, and video_S3_Ez_1650nm

\section{Title: On-chip zero-index metamaterials}

\author{
Authors: Yang $\mathrm{Li}^{2} \uparrow$, Shota Kita ${ }^{1} \uparrow$, Philip Muñoz ${ }^{1}$, Orad Reshef ${ }^{1}$, Daryl I. Vulis ${ }^{1}$, Mei Yin ${ }^{1,2}$, \\ Marko Lončar ${ }^{1 *}$, and Eric Mazur ${ }^{1,3 *}$
}

\footnotetext{
${ }^{2}$ School of Engineering and Applied Sciences, Harvard University, 9 Oxford Street, Cambridge, Massachusetts 02138, USA.

${ }^{2}$ State Key Laboratory of Advanced Optical Communication Systems and Networks, School of Electronics Engineering and Computer Science, Peking University, 5 Yiheyuan Road, Beijing 100871, China.

${ }^{3}$ Department of Physics, Harvard University, 9 Oxford Street, Cambridge, Massachusetts 02138, USA.

$\dagger$ These authors contributed equally to this work.

*e-mail: 1oncar@seas.harvard.edu; mazur@seas.harvard.edu
} 


\section{S.1 Prism design}

In the design of the metamaterial prism, we consider two factors: homogenity and transmission. As shown in Fig. S1, the far-field pattern shows a narrower refracted beam and weaker side beams as the size of the prism increases. The reason is that the metamaterial prism can be better treated as a homogenous-bulk prism if it consists of more unit cells (Section S.5). Fig. S2 shows that the transmission of the prism decreases as its size increases. This is because the total propagation loss and in-plane radiation loss are proportional to the size of the prism. For a finite metamaterial prism, the total losses include not only the coupling and propagation losses as well as the loss resulting from coupling of energy to side bands (Section S.4), but also the in-plane radiation loss due to the radiation of the non-output facets. Thus, there is a trade-off between a better homogenity and a high transmission. To guarantee that sufficient power can reach the numerical aperture of the microscopy and a distinguishable refracted beam in the far-field pattern, we choose a prism consisting of $8 \times 8$ pitches.

\section{S.2 Effect of gold structures}

The zero-index effect that is demonstrated in this work is due to Mie resonances of a purely dielectric photonic crystal ${ }^{17}$. Though we incorporate gold structures into our design, these metal layers only contribute to the enforcement of the fundamental TM mode, making the short pillars optically infinite tall, and the confinement of the light within the metamaterial structure; they do not make any plasmonic resonance-based contribution to the metamaterial. In this section, we show that there exist Dirac cones without any metal and that the metal only contributes to the 
overall performance of the metamaterial. The different contributions of the gold mirrors and the gold caps above the pillars are investigated separately below.

\section{A. Gold mirrors}

We simulate the modes at the $\Gamma$ point for our proposed structure without the gold mirrors. By tuning the thickness of the SU-8 polymer, as discussed in section S.6, we obtain an additional degree of freedom by which we can engineer a degeneracy at the $\Gamma$ point, even without the gold mirrors. By tuning the thickness of the SU-8 polymer layer, we obtain multiple geometries that exhibit the degeneracy between the monopole and dipole modes that is necessary to obtain a Dirac cone. These correspond to SU-8 thicknesses of $687.5 \mathrm{~nm}, 1125 \mathrm{~nm}, 1575 \mathrm{~nm}$, and $1725 \mathrm{~nm}$.

We calculate the band structures for these geometries to verify their degeneracies (Fig. S3). In the absence of the gold mirrors, the resulting band structures are less linear and more quadratic near the degeneracy point. However, we still observe a conical shape and a degeneracy at a single wavelength, indicative of a Dirac-cone dispersion.

We estimate the propagation loss for the structures with the modal degeneracies using finite-difference time-domain (FDTD) simulations and the method described in section S.4. The resulting propagation loss are plotted in Fig. S4. We also plot the propagation loss for the corresponding gold-mirror Dirac-cone structures for comparison. We obtain lower propagation loss when using the gold mirrors. Given the shape of the Dirac cones and the propagation loss that are obtained using the structures that are cladded with gold, it is clear that the performance of the metamaterial is better when using the gold mirrors. This is due to the additional confinement 
provided by the mirrors as well as due to the enforcement of a clean TM mode by constraining the electric field to be normal to the parallel plates.

\section{B. Gold caps}

Due to the fabrication procedure, the lower gold mirror in the structure creates a cap on top of the silicon pillars upon deposition. This cap is only a consequence of the fabrication procedure, and not a necessary component of the metamaterial. We demonstrate this by simulating the band structure of the proposed structure without these gold caps and also producing a Dirac-cone dispersion.

Removing the gold cap causes effects analogous to a deviation from the ideal radius described in section S.12. We run the optimization scheme described in section S.3 to obtain new

dimensions for the smallest possible $\left|n_{\text {eff }}\right|$, with a radius of $189 \mathrm{~nm}$ and a pitch of $727 \mathrm{~nm}$. We simulate the band structure for this geometry and display it in Fig. S5. We once again obtain the degeneracy between the monopole mode and the dipole modes at the $\Gamma$ point. Additionally, because this hypothetical design still possesses the gold mirrors, the Dirac cone it exhibits is as linear as those showed in Figure S15b that also have the gold caps. Thus, the gold caps do not play an important role in the demonstrated zero-index behavior.

\section{S.3 Metamaterial design}

Dirac-cone metamaterials can be formed using square- or triangular-lattice photonic crystals $^{17}$. We choose the square lattice because it brings the advantage of well-defined metamaterial boundaries. We initially design the pitch $a$ and radius $r$ of the Dirac-cone metamaterial using an analytical model for the effective constitutive parameters of a square array of $2 \mathrm{D}$ magnetodielectric cylinders ${ }^{25}$. Based on this result and the given values of height of the 
silicon pillars, the SU-8 layer thickness, and the gold layer thickness, we optimize $a$ and $r$ based on a 3D FDTD model of the presented structure (Fig. 1a). We choose $a$ and $r$ as the optimization parameters since the existence of the Dirac cone is most sensitive to these two geometric parameters. The figure of merit of this optimization is the absolute value of the effective index $\left|n_{\text {eff }}\right|$, which is retrieved from the simulated reflection and transmission coefficients, at the design wavelength, $\lambda=1590 \mathrm{~nm}$. This figure of merit is selected in order to design a Dirac-cone metamaterial with zero real index and low propagation loss, the latter of which can be characterized by the imaginary part of the refractive index at the design wavelength. As shown in Fig. S6a and $\mathrm{b}$, the minimum values of the real and imaginary parts of $n_{\text {eff }}$ appear along the two different diagonals of the 2D parameter space, and cross each other around the center. Hence, the minimum value of $\left|n_{\text {eff }}\right|\left(n_{\text {eff }}=0.002+i 0.046\right)$ appears around the center of the $2 \mathrm{D}$ parameter space at $a=690 \mathrm{~nm}$ and $r=211 \mathrm{~nm}$ (Fig. S6c). These values combined with other geometric parameters in Fig. 1a are used as the target parameters of our nanofabrication.

Since the existence of a Dirac cone is sensitive to the geometric parameters of the structure, it is important to investigate the robustness of our design with regards to the effects induced by parameter variations. Here, we study the effects of variations in the pillar radius $r$ (Fig. 1a) since this parameter represents the largest source of disorder among all the geometric parameters. We model the effect of disorder on the transmission through the structure assuming a Gaussian distribution with a mean radius $r=211 \mathrm{~nm}$ and standard deviation $\Delta r$. Using 3D FDTD simulations, we test the transmission through a metamaterial slab (Fig. 1a) with 8 unit cells in the direction of propagation, which is the number of periods in our fabricated prism sample (Fig. 1b, c). As depicted in Fig. S7, this metamaterial shows transmission over $10 \%$ at $1590 \mathrm{~nm}$ until the 
deviation of radius is as large as $\Delta r / r=6 \%$, which is within the fabrication tolerance we can achieve $( \pm 5 \%)$.

\section{S.4 Losses}

Losses in the metamaterial originate from three major sources: (i) coupling loss at the interface due to impedance mismatch; (ii) propagation loss within the metamaterial; and (iii) coupling of energy to side bands (Section S.9).

We estimate both the coupling and propagation losses of the metamaterial using an FDTD simulation. In this simulation, we couple into and out of a transversely infinite array of pillars using a pair of 500-nm-thick silicon slab waveguides. The fundamental TM mode is launched in the input slab waveguide and the transmission of $E_{z}$ is monitored in the output waveguide. We increase the length of the metamaterial in the direction of propagation and plot the transmission as a function of the number of unit cells (Fig. S8). The intercept located at $y=-2.8 \mathrm{~dB}$ corresponds to the input and output coupling losses to the waveguides. This means that the coupling loss at a single interface is $1.4 \mathrm{~dB}(28 \%)$. To further minimize this loss, we can engineer either or both the impedance of the coupling structure and that of the metamaterial. Impedance of the coupling structure can be engineered in various ways, such as a photonic-crystal-waveguide taper ${ }^{26}$. To tune the effective impedance of the metamaterial while keeping the absolute effective index closes to zero (Section S.3), we need an additional degree of freedom besides the radius and pitch. For example, we can tune the height of the pillars of an on-chip all-dielectric zero-index metamaterial consisting of square array of silicon pillars on a silicon-on-insulator (SOI) substrate ${ }^{27}$. We also extract the propagation loss of the metamaterial, which is $0.9 \mathrm{~dB} /$ unit cell $(1.3 \mathrm{~dB} / \mu \mathrm{m})$. We 
believe this loss can be further minimized by cladding our pillar structures with a lower-loss mirror such as a Bragg stack ${ }^{28}$ or by converting the accessed modes into bound states in continuum ${ }^{29}$.

We estimate the loss due to coupling of energy to side bands by investigating the refracted and side beams (Section S.9) of our metamaterial (Section S.3) in the FDTD simulated far-field pattern (Section S.10). At the input facet of the metamaterial prism (Fig. 3b), the fundamental TM mode in the silicon waveguide excites the main bands (the two linear bands that form the Diraclike cone, Fig. 2a, b) and part of the side bands (Fig. 2a). At the output facet of the prism, the main and side bands contribute to the refracted and side beams, respectively, which propagate in the SU-8 slab waveguide and eventually reach the far field (Fig. 3b). Thus, we can estimate the energy ratio of the main bands by evaluating that of the refracted beam in the far-field pattern. As shown in Figure $\mathrm{S} 9$, at $1590 \mathrm{~nm}$, the refracted beam of a $10 \times 10$ prism points in the $\theta=0^{\circ}$ direction and contains the maximum electric field intensity. We obtain the energy ratio of the refracted beam by the division of the total electric field intensity within the beam width of the refracted beam by the total electric field intensity in the angular range from $-90^{\circ}$ to $90^{\circ}$. Here, we define the beam width of the refracted beam as its first-null beam width ${ }^{30},-21.3^{\circ}$ to $16.8^{\circ}$ (Fig. S9). At $1590 \mathrm{~nm}$, the energy ratio of the refracted beam (main bands) is $36 \%$, corresponding to an additional loss of $-4.4 \mathrm{~dB}$. This ratio for prisms with sizes $5 \times 5,15 \times 15$, and $20 \times 20$ are $44 \%(-3.6 \mathrm{~dB}), 40 \%$ ( $-4.0 \mathrm{~dB})$, and $46 \%(-3.4 \mathrm{~dB})$, respectively (Fig. S1). We can see that this ratio varies insignificantly as a function of the prism size. Using topology optimization, we could design a better coupling structure that only excites the main bands ${ }^{31,32}$.

\section{S.5 Homogenization}

We conduct a systematic homogenization analysis to verify that it is proper to treat the onchip Dirac-cone metamaterial macroscopically as a homogeneous bulk medium with effective 
constitutive parameters in the in-plane direction near the $\Gamma$-point. In this analysis, we consider the homogenization criterion and locality conditions. Furthermore, we compare our Dirac-cone metamaterial with its corresponding homogenized model. Since our structure (Fig. 1a) is only periodic in the in-plane direction, this analysis is restricted to in-plane propagation. In this paper, we assume harmonic time dependence of $\mathrm{e}^{-i \omega t}$.

\section{A. Homogenization criterion}

The general homogenization criterion of metamaterials requires that $k_{0} a, k a \leq 1$, where $a$ is the lattice constant (Fig. 1a), $k_{0}$ and $k$ are the free-space wavenumber and the effective wavenumber in the metamaterial, respectively ${ }^{33}$. Based on this criterion, we analyze the homogenization of our metamaterial within the array and at the boundary, separately.

Within the array, the metamaterial can be treated as an infinite array, which can be analyzed by studying its photonic band structure. Considering that the Dirac cone appears near the $\Gamma$-point (Fig. 2a) where the effective wavenumber $k$ approaches zero, this metamaterial meets the criterion $k a \leq 1$ in the vicinity of the Dirac point. To quantitatively estimate the region, in which the effective constitutive parameters are valid, we compare band structures obtained using two different methods: a macroscopic method treating the metamaterial as a homogeneous bulk medium (using the retrieved effective index $n_{\text {eff }}$ and the expression $k=n_{\text {eff }} \omega / c$, and a microscopic method regarding the metamaterial as an infinite array (FDTD). As shown in Fig. $\mathrm{S} 10$, the two band structures agree well in the range $0<k<0.3$ for the linear band below the Dirac point and in the range $0<k<0.4$ for the linear band above the Dirac point. Thus, it is reasonable to treat the infinite array as a homogeneous bulk medium with effective constitutive parameters within these regions. Further, because our metamaterial is almost isotropic near the Dirac point 
(Fig. 2d), this conclusion is valid for all propagation directions near the Dirac point in the Brillouin zone.

Because the lattice constant of our Dirac-cone metamaterial is not much smaller than the free-space wavelength $\left(k_{0} a=2.73\right)$, the homogenization criterion $k_{0} a \leq 1$ is not met and we cannot define the physical boundary of this metamaterial unambiguously. Instead, there is a transition layer in which the local effective constitutive parameters vary from the values of the infinite array to the values of the background medium surrounding the metamaterial ${ }^{34}$. To investigate this transition layer quantitatively, we use 3D FDTD to compare the phase of the electric field at the interface of our Dirac-cone metamaterial with that of a corresponding bulk zero-index medium with retrieved constitutive parameters (Fig. 2e). Here, we analyze the phase instead of the local effective constitutive parameters due to following reasons: first, phase can directly reflect the variation in the local constitutive parameters; second, it's challenging to extract the local effective constitutive parameters at the interface. To observe the change in the phase near the boundary clearly, we position the SU-8 background medium, metamaterial, and its homogenized model between gold layers (Fig. S11a). This guarantees an efficient coupling between the TM mode in the input waveguide and that in the metamaterial/homogenized model. Within the metamaterial, we only consider the phase of the electric field in-between the pillars since the local effective constitutive parameters are a consequence of the interaction of the electromagnetic fields scattered by the pillars in the array. As shown in Fig. S11b and c, the behavior of the phase at the interface of the metamaterial is very similar to that of the homogenized model. Fig. S11d illustrates this agreement more clearly: the phase advance is essentially linear along the propagation direction in the positive-index input waveguide. However, we observe uniform phase within the metamaterial/homogenized-model due to their zero indices. The 
transition between these two behaviors happens very sharply at the interface, indicating a discontinuity in the local constitutive parameters at the boundary of the metamaterial. In other words, similar to its homogenized model, our metamaterial also has a well-defined boundary in terms of local constitutive parameters even though its lattice constant is not much smaller than the free-space wavelength. The minor phase oscillation in the input waveguide is due to a standing wave consisting of the incident traveling wave and the wave reflected by the metamaterial or its homogenized model, and the phase oscillation in the metamaterial regime is due to the interaction of the electromagnetic fields scattered by the pillars in the array. As for the phase of the electric field within one unit cell of the metamaterial, we would like to clarify that the mode continuously oscillates between an electric-monopole mode and a transverse-magnetic-dipole mode (see animations: video_S1_Ez_1530nm, video_S2_Ez_1590nm, and video_S3_Ez_1650nm). However, the phase advance remains zero between two same positions in different unit cells. (Fig. S11b, top).

To further investigate the homogenization at the boundary, we simulate the angledependent transmission of both our Dirac-cone metamaterial and its homogenized model. We calculate the transmission through a metamaterial slab with a thickness of five unit cells (along the propagation direction) and an identically sized bulk medium (Fig. 1a). Fig. S12 shows the transmission at incident angles ranging from $0^{\circ}$ (normal incidence) to $80^{\circ}$ at the design wavelength of $1590 \mathrm{~nm}$. This range was selected to avoid artifacts in transmission for angles of incidence greater than $80^{\circ}$.

We observe high transmission near normal incidence for both cases, indicating strong angular selectivity and good agreement between the metamaterial and homogenized model. Considering the critical angle of a material with an index of zero, transmission at only normal 
incidence is expected. Extraneous transmission at higher angles of incidence, as observed in the case of the metamaterial, can be attributed to incident light coupling into the longitudinal magnetic dipole mode crossing the Dirac point as a quadratic band (Fig. 2b). This mode can only be excited when the incident light has non-zero k-components parallel to the interface ${ }^{17}$. This extraneous transmission is reduced at wavelengths less than the Dirac-point wavelength of $1590 \mathrm{~nm}$, as the longitudinal mode does not exist in this regime (Fig. S12a, Fig. 2b). We also observe a decrease in angular selectivity for both the metamaterial and bulk medium at lower wavelengths since the indices of both cases are deviating from zero in this regime.

\section{B. Locality conditions}

Effective constitutive parameters of metamaterials have to satisfy the conditions of locality: passivity, causality, isotropy, and absence of radiation $\operatorname{loss}^{34}$. Most metamaterials cannot meet the fourth condition since the size of the resonator is not much smaller than the wavelength so that there is scattering from single inclusions. Fig. S13 shows that the medium is passive, since $\operatorname{Im}\left(\varepsilon_{\mathrm{r}}^{\text {eff }}\right)>0$ and $\operatorname{Im}\left(\mu_{\mathrm{r}}^{\text {eff }}\right)>0$ simultaneously near the Dirac-point wavelength. As shown in Fig.

2e, the fact that both $\partial\left(\operatorname{Re}\left(\varepsilon_{\mathrm{r}}^{\text {eff }}\right)\right) / \partial \omega>0$ and $\partial\left(\operatorname{Re}\left(\mu_{\mathrm{r}}^{\text {eff }}\right)\right) / \partial \omega>0$ near the Dirac-point wavelength indicates that the medium satisfies the basic causality conditions represented by the KramersKronig relations ${ }^{35}$. The isotropy of our metamaterial in the vicinity of the Dirac point has already been verified in Fig. 2d. Similar to most metamaterials, our metamaterial does not satisfy the fourth 
condition since its inclusion size is not much smaller than the free-space wavelength so that the scattering loss cannot be ignored.

\section{Comparison of Dirac-cone metamaterial prism with its homogenized model}

We compare the FDTD simulations of the prism composed of the Dirac-cone metamaterial (Fig. 1a) with its corresponding homogenized model using the retrieved effective constitutive parameters at the designed zero-index wavelength $1590 \mathrm{~nm}$ (Fig. 2e). As shown in Fig. S14, both prisms show good transmission at the input interface due to their finite characteristic impedance 1.47. Within both prisms, the electric fields exhibit almost uniform distribution due to the nearzero real indices of the prisms $\operatorname{Re}\left(n_{\text {eff }}\right)=1.9 \times 10^{-3}$. The fields decay along the propagation direction, which is normal to the input interface, due to the propagation losses of the prisms $\operatorname{Im}\left(n_{\text {eff }}\right)=4.6 \times 10^{-2}$. In the SU-8 slab waveguide region, both prisms show the refracted beam normal to the output interface. Unlike the metamaterial, the homogenized-model prism shows a refracted beam without any side beams since no side bands exist in the band structure of the homogenized model (Section S.9).

\section{S.6 Dirac cones of metamaterials with large SU-8 thickness}

Here we analyze the effect of variations in SU-8 thickness on the existence of a Dirac cone, especially for thicknesses much larger than height of the silicon pillars (Fig. 1a).

The Dirac cone is a consequence of the degeneracy of an electric monopole mode and a transverse magnetic dipole mode at the $\Gamma$ point, so any variation in the wavelengths of these modes at the $\Gamma$ point would adversely affect the existence of the Dirac cone. Fig. S15a shows the $\Gamma$ point wavelengths of these modes for various SU-8 thicknesses. As the SU-8 thickness increases from $500 \mathrm{~nm}$ to $2 \mu \mathrm{m}$, the electric monopole mode at the $\Gamma$ point remains nearly unchanged around 
$1590 \mathrm{~nm}$ while there are four different magnetic dipole modes at the $\Gamma$ point, $\mathrm{d}_{\mathrm{i}-\mathrm{iv}}$. Each of these dipole modes intersects with the electric monopole mode at a particular SU-8 thickness, which indicates the existence of a Dirac cone. To confirm this fact, we calculated the band structure near the $\Gamma$ point with SU-8 thicknesses corresponding to each of those intersections (Fig. S15b). Results clearly show the existence of Dirac cones when SU-8 thickness equals to $550 \mathrm{~nm}, 975 \mathrm{~nm}$, $1475 \mathrm{~nm}$, and $1975 \mathrm{~nm}$, respectively.

Although our structure shows Dirac cones with different values of SU-8 thickness, we still need to verify that those Dirac cones are due to the silicon pillars rather than metallic waveguides, especially when the SU-8 thickness is much larger than the height of the pillars. We analyze the mode profiles of the two modes forming the Dirac cone by observing the spatial distribution of the out-of-plane electric field component $E_{z}$ in a single unit cell. As depicted in Fig. S16, for all SU8 thickness showing Dirac cones, the fields of both the electric monopole mode and the transverse magnetic dipole mode are confined within the pillar region. This confirms that the Dirac cones corresponding to structures with different SU-8 thicknesses (Fig. S15) are all due to the interaction between the electromagnetic wave and the array of pillars which form the patterned metamaterial, rather than the metallic waveguide.

Our fabricated Dirac-cone metamaterial prism (Fig. 1b, c) has an SU-8 thickness around $1500 \mathrm{~nm}$, instead of the initially designed value of $595 \mathrm{~nm}$ (Fig. 1a). This thickness corresponds 
to intersection C in Fig. S15, between the third order transverse magnetic dipole mode $d_{i i i}$ and the electric monopole mode m.

\section{S.7 Modes in SU-8 slab waveguide}

In Fig. S17a (Fig. 3b), we observe a low-frequency oscillating pattern in the SU-8 slab waveguide at the prism output. We claim that this is due to the interference between the multiple propagating TM modes in the SU-8 planar waveguide. We prove that this is the origin of the lowfrequency oscillating pattern using two methods.

First, we solve Helmholtz equation for an asymmetric slab waveguide ${ }^{36}$. In the case of a 2$\mu$ m-thick SU-8 slab on a silica substrate in air, the solution at $\lambda=1.57 \mu \mathrm{m}$ yields a pair of TM modes with effective indices 1.5372 and 1.4526. These indices correspond to effective wavelengths of 1.021 and $1.080 \mu \mathrm{m}$, respectively. We can combine two beams to obtain a beatwavelength of:

$$
\lambda_{\text {beat }}=\left(\frac{1}{\lambda_{\text {eff }}^{(1)}}-\frac{1}{\lambda_{\text {eff }}^{(2)}}\right)^{-1}=18.5 \mu \mathrm{m}
$$

This is precisely the spatial extent of the oscillating patterns shown in Fig. S17a.

Second, we plot the electric field of the refracted beam along the propagation direction (Fig. S17c), as well as its Fourier transform (Fig. S17d), to reveal the dominant spatial frequencies. Two peaks are apparent at the spatial frequencies of 0.9239 and $0.9828 \mu^{-1}$, corresponding to effective wavelengths of 1.082 and $1.018 \mu \mathrm{m}$. These wavelengths match the results found in the first method for the two propagating TM modes in the planar waveguide.

Finally, we simulate an identical prism structure followed by a thinner SU-8 planar waveguide, which only supports one propagating TM mode. Numerical calculations show that the 
low-frequency oscillating pattern has been suppressed (Fig. S17b), confirming our claim that the oscillation is due to the interference between the multiple propagating TM modes.

\section{S.8 Experimental results near zero-index wavelength}

To illustrate the refractions of our metamaterial prism near its zero-index wavelength, we show the experimental results at 1480 and $1620 \mathrm{~nm}$ (Fig. S18). As indicated by the yellow arrow, the refracted beam propagates toward a positive and a negative refraction angle, respectively, at 1480 and $1620 \mathrm{~nm}$. We can also observe several side beams at those wavelengths.

\section{S.9 Side beams}

The side beams in Fig. 3 and Fig. S20 are due to coupling to the bands which appear near the Dirac-cone frequency regime but do not form the Dirac cone. Here, we call those bands "side bands". Due to the gold layers and the finite thickness of the pillars, multiple side bands (Fig. 2a) exist in the band structure of our metamaterial (Fig. 1a). To clearly investigate the relationship between the side bands and the side beams, we choose to analyze a simplified version of our metamaterial - a 2D square array of infinitely long silicon pillars in an SU-8 matrix (Fig. S19 a). We design this 2D metamaterial with a zero index at $1550 \mathrm{~nm}(193.55 \mathrm{THz})$. Its band structure shows a Dirac-cone dispersion at the $\Gamma$ point and at $193.55 \mathrm{THz}$ there only a few side bands (Fig. S19 c, d). By comparing Fig. S19 b, c, and d, we can see that the linear dispersion bands from 180 to $\sim 202 \mathrm{THz}$ near the $\Gamma$ point correspond to the refracted beam continuously changing from $-6^{\circ}$ at $180 \mathrm{THz}$ to $6^{\circ}$ at $\sim 202 \mathrm{THz}$. Due to the fact that there is no side bands above the SU-8 light line in this frequency regime and the fact that the quadratic band crossing the Dirac point cannot be excited by the fundamental TM mode of the input waveguide, there is no side beam in this frequency regime (Fig. S19 b). In the frequency regime higher than $\sim 202 \mathrm{THz}$, two side bands 
appear on both sides of the M point above the SU-8 light lines, of which the side band in $\mathrm{M}-\Gamma$ is much stronger than that in the X-M (Fig. S19c, d). We believe that the side band in $\mathrm{M}-\Gamma$ corresponds to the side beam continuously varying from $-70^{\circ}$ at $\sim 202 \mathrm{THz}$ to $-58^{\circ}$ at $210 \mathrm{THz}$ (Fig. S19 b). Because this side band is also stronger than the linear bands near the $\Gamma$ point, its corresponding side beam is stronger than the refracted beam. We also note that the refracted beam shows a gap at $\sim 202 \mathrm{THz}$. It is due to the fact that most power at this frequency is shifted from the linear bands to the side band in $\mathrm{M}-\Gamma$.

Because the period of the pillar array $(690 \mathrm{~nm})$ is not much smaller than the effective wavelength in the SU-8 slab waveguide (1033 nm at the design wavelength of $1590 \mathrm{~nm}$ ), we also consider the effects of diffraction at the interface between the prism and the SU-8 slab waveguide ${ }^{37}$ (Fig. 1). This diffraction is described by the grating equation, $d \sin 45^{\circ} / \lambda_{\text {eff }}+d \sin \theta_{m} / \lambda_{\text {eff2 }}=m$, where $d$ is the spacing between neighboring silicon pillars along the interface $(\sqrt{2} a$, where $a$ is the period of the silicon pillar arrays as shown in Fig. 1a), $\theta_{m}$ is the angle between the diffracted ray and the surface normal, $m$ is the order of the diffracted mode, $\lambda_{\text {effl }}$ and $\lambda_{\text {eff2 }}$ are the effective wavelengths in the prism and the SU-8 slab waveguide, respectively. In our case, $\theta_{ \pm 1}=\sin ^{-1}(1033 /(\sqrt{2} \cdot 690))=90^{\circ}-19.52^{\circ} \mathrm{i}$, indicates that the output from the prism only includes a zeroth-order beam, which is the refracted beam obeying Snell's law, without any diffraction. However, diffraction will appear if the mode index of the output slab waveguide is larger than 1.63. In practical integrated-photonics applications, the diffractions can be suppressed by aligning 
the output facet to the $\Gamma-\mathrm{X}$ orientation, by decreasing the mode indices of the input and output waveguides, or by adiabatically tapering down the pillar spacing at the boundaries.

\section{S.10 Far-field patterns with different normalizations}

In Fig. 3c, we show the far-field patterns normalized at each wavelength in the refractionangle range of $-45^{\circ} \leq \alpha \leq 45^{\circ}$. Here, we show the far-field patterns normalized to the global maximum and at each wavelength, respectively, in the refraction-angle range of $-90^{\circ} \leq \alpha \leq 90^{\circ}$. As shown in Fig. S20a, both measured and FDTD far-field patterns show that most of the energy goes to the refracted beam around the zero-index wavelength, $1570 \mathrm{~nm}$. This is because we optimize our metamaterial to exhibit both a real index of zero and low propagation loss at the design wavelength (Section 3). Fig. S20b shows that one side beam shifts from $-75^{\circ}$ at $1580 \mathrm{~nm}$ to $-60^{\circ}$ at $1480 \mathrm{~nm}$ while the other side beam shifts from $40^{\circ}$ at $1680 \mathrm{~nm}$ to $45^{\circ}$ at $1643 \mathrm{~nm}$.

\section{S.11 Prism index extraction and error estimation}

We extract the prism index from InGaAs images of refraction through a zero-index metamaterial prism. Figs. 1b and S21a show a silicon waveguide feeding into a triangular prism, which couples to a large SU-8 slab waveguide with a radius of $125 \mu \mathrm{m}$. Light enters through the silicon waveguide and refracts through the prism into the SU-8 slab waveguide. This beam scatters at the edge of the SU-8 slab waveguide, and is imaged from above using an InGaAs camera (Fig. S21b). Images are taken for a range of input wavelengths between $1480 \mathrm{~nm}$ and $1680 \mathrm{~nm}$. In addition to refraction images, we use reference images to identify alignment marks, which are used to locate and orient the prism.

We first determine the position of the alignment marks in the microscope images using a 2D Gaussian fit. This allows us to orient the prism and map pixels near the edge to polar 
coordinates. The refracted beam is then located using a polar 2D Gaussian fit to extract the refracted angle. This refracted angle is used to calculate the prism index using Snell's law and the effective index of the SU-8 slab waveguide. The measurement error is propagated throughout the calculation and includes the effects of camera pixel size, resolution, and fit uncertainty due to variance in the data.

\section{A. Alignment marks}

There are two alignment marks placed at $\pm 45^{\circ}$ relative to the surface normal of the prism output, and 139.2 $\mu \mathrm{m}$ from the center of the prism (Fig. S21a). They are both $3 \mu \mathrm{m}$ wide, and show up brightly in the images as shown in Fig. S22a. By identifying the locations of each alignment mark we can determine the SU-8 slab waveguide's radius $r$, rotation offset $\alpha_{\circ}$, and prism location from which we define the origin $\left\{x_{\circ}, y_{\circ}\right\}$ :

$$
\begin{gathered}
r=\frac{125 \mu \mathrm{m}}{139.2 \mu \mathrm{m}} \sqrt{\frac{\left(x_{\mathrm{AM} 1}-x_{\mathrm{AM} 2}\right)^{2}+\left(y_{\mathrm{AM} 1}-y_{\mathrm{AM} 2}\right)^{2}}{2}} \\
\alpha_{\circ}=\tan ^{-1}\left(\frac{y_{\mathrm{AM} 1}-y_{\mathrm{AM} 2}}{x_{\mathrm{AM} 1}-x_{\mathrm{AM} 2}}\right) \\
\left\{\begin{array}{l}
x_{\circ}=x_{\mathrm{AM} 1}+\frac{139.2 \mu \mathrm{m}}{125 \mu \mathrm{m}} r \cos \left(\frac{\pi}{4}+\alpha_{\circ}\right) \\
y_{\circ}=y_{\mathrm{AM} 1}+\frac{139.2 \mu \mathrm{m}}{125 \mu \mathrm{m}} r \sin \left(\frac{\pi}{4}+\alpha_{\circ}\right)
\end{array}\right.
\end{gathered}
$$

where the measured values $\left\{x_{\mathrm{AM} 1}, y_{\mathrm{AM} 1}\right\}$ and $\left\{x_{\mathrm{AM} 2}, y_{\mathrm{AM} 2}\right\}$ are the coordinates of the two alignment marks.

To determine the center of the alignment marks, we fit the intensity profile of each mark to a $2 \mathrm{D}$ Gaussian function: 


$$
I\left(x, y ; a, b_{x}, b_{y}, c, d\right)=a \cdot \mathrm{e}^{-\left[\frac{\left(x-b_{x}\right)^{2}}{c^{2}}-\frac{\left(y-b_{y}\right)^{2}}{c^{2}}\right]}+d
$$

The fitting parameters are amplitude $a, x$-center position $b_{x}, y$-center position $b_{y}$, width $c$, and intensity offset $d$. We use a nonlinear least-squares optimization method to minimize the residuals of the data (Fig. S22b). This solution also includes an estimate of the covariance of the fitting parameters.

To ensure accurate fits for the data, we consider only the pixels in the vicinity of the alignment marks. Too narrow a window would reduce the fit quality, and too wide a window would include other peaks. We use a 20-pixel-wide window, which is significantly wider than the alignment mark $(c=1.38 \mathrm{px})$, but small enough to exclude extraneous features. The window is centered at the maximum intensity for each alignment mark. From the fit, we calculate the SU-8 slab waveguide radius $r$, offset angle $\alpha_{\circ}$ and the origin position $\left\{x_{\circ}, y_{\circ}\right\}$.

\section{B. Refraction angle}

From the measured positions of the alignment marks, we can define the edge of the SU- 8 slab waveguide. The refracted beam propagating along the SU-8 slab waveguide will be scattered from this edge, appearing as a bright band. Since the edge is curved, it is convenient to map the positions of the pixels to polar coordinates centered at the prism (Fig. S23b). We can then locate the center of the refracted beam using the same method as for the alignment marks.

Pixels are mapped to polar coordinates $\{x, y\} \rightarrow\{r, \alpha\}$ according to the alignment mark measurements:

$$
\left\{\begin{array}{c}
r=\sqrt{\left(x-x_{\circ}\right)^{2}+\left(y-y_{\circ}\right)^{2}} \\
\alpha=\tan ^{-1}\left(\frac{y-y_{\circ}}{x-x_{\circ}}\right)+\alpha_{\circ}
\end{array}\right.
$$


In polar coordinates, we fit the beam intensity profile to another 2D Gaussian, this time allowing for two peaks to account for the sometimes-irregular beam profile:

$$
I\left(r, \alpha ; a_{1}, a_{2}, b_{r}, b_{1 \alpha}, b_{2 \alpha}, c_{r}, c_{1 \alpha}, c_{2 \alpha}, d\right)=a_{1} \cdot \mathrm{e}^{-\left[\frac{\left(r-b_{r}\right)^{2}}{2 c_{r}^{2}}+\frac{\left(\alpha-b_{1 \alpha}\right)^{2}}{2 c_{1 \alpha}}\right]}+a_{2} \cdot \mathrm{e}^{-\left[\frac{\left(r-b_{r}\right)^{2}}{2 c_{r}^{2}}+\frac{\left(\alpha-b_{2 \alpha}\right)^{2}}{2 c_{2 \alpha}}\right]}+d
$$

The refracted angle is measured using the stronger of the two peaks. Fig. S23d shows an example of the 2D Gaussian fit for an excitation wavelength of $1590 \mathrm{~nm}$. We repeat this measurement for several wavelengths to track the refracted beam.

\section{Prism index}

The prism index is calculated using the measured refraction angle. Light enters the prism normal to the input surface, and refracts into the SU-8 slab waveguide at an incident angle of $45^{\circ}$. From Snell's law:

$$
n_{\text {prism }}=n_{\text {slab }} \frac{\sin \left(\alpha_{\mathrm{c}}\right)}{\sin \left(45^{\circ}\right)}=1.54 \sqrt{2} \sin \left(\alpha_{\mathrm{c}}\right)
$$

The extracted index shows a continuous transition from positive to negative index with increasing wavelength (Fig. 3d). The zero crossing is centered on $1570 \mathrm{~nm}$, where the index dispersion is linear. The error bars in this plot represent the uncertainty in the measured index, assuming a confidence level of $95 \%{ }^{38}$. They include the effects of the image size, pixel size, resolution, and propagated errors from each measurement stage. Given the calculated uncertainty in the measurement, the observed zero index "bandgap", in which the real index is equal to zero with $95 \%$ confidence, is at most $49 \mathrm{~nm}$ wide.

\section{S.12 Change in Dirac cone with different pillar radii}

To verify that the measured zero index is due to the existence of a Dirac cone, we simulate and fabricate a prism with pillar radii that are larger than that of the prism in Fig. 3, $r=190 \mathrm{~nm}$. 
For this prism, we compare the band structure as well as the measured and simulated indices to the ideal case. The simulated band structure only exhibits a Dirac cone for the ideal radius $r=190 \mathrm{~nm}$ ; otherwise, a bandgap appears between the electric monopole mode and the transverse magnetic dipole modes (Fig. S24a, c). When the radius deviates from the ideal case, the real part of the index is fixed at zero over a range of wavelengths between the positive and negative index regimes, indicating a bandgap. From an effective medium perspective, changing the pillar radius breaks the degeneracy of the magnetic and electric plasma frequencies, resulting in a frequency range in which either the effective permittivity or effective permeability is negative, but not both.

The band structure for a metamaterial with larger $r$ shows dipole modes at the $\Gamma$ point that are redshifted, so as to open a bandgap in the wavelength regime of 1570 to $1610 \mathrm{~nm}$ (Fig. S24a). This fact is clearly verified by the measured and simulated $n_{\text {eff }}$ shown in Fig. S24b: the zero crossing of the index is redshifted, and the measured index remains at zero throughout the bandgap region. In simulations, the refracted beam is relatively weak for these wavelengths, resulting in relatively large fitting uncertainty within the bandgap. Here, we plot the simulated index with a dashed line when the uncertainty is larger than the average uncertainty over the entire bandwidth. Considering the error bars, the measured bandgap is at most $95 \mathrm{~nm}$ wide, centered at $1568 \mathrm{~nm}$ (from 1520 to $1615 \mathrm{~nm}$ ), which is $94 \%$ wider than that of the prism with ideal radius.

These results confirm the importance of degeneracy of the monopole and dipole modes, and establish a connection between predicted band structure and prism index. We show that the experimentally measured zero index of our metamaterial with the ideal radius of $r=190 \mathrm{~nm}$ corresponds to a Dirac cone at the $\Gamma$ point. 


\section{S.13 Control experiment}

To validate the results of the metamaterial prism index measurement, we perform a control measurement on the same measurement setup (Fig. 1b, c) without the prism. In this control device, light from the input silicon waveguide couples directly into free space $\left(n_{\text {air }}=1\right)$ before entering the SU-8 slab waveguide at an incident angle of $45^{\circ}$ (Fig. S25a). Using the same experimental and simulation methods as the prism measurement, we observe a refracted beam in the far field at an angle of $25.61^{\circ} \pm 1.32^{\circ}$ (Fig. S25b). Based on the Snell's law, this angle corresponds to refraction from a material with index $n=0.94 \pm 0.04$, which is very close to the index of air. Fig. S25c shows the measured and simulated indices of the control experiment, which agree with each other well throughout the measurement wavelength range.

These results confirm that the measured index shown in Fig. 3 corresponds to the effective index of the metamaterial prism, instead of the artifact of the measurement setup. 

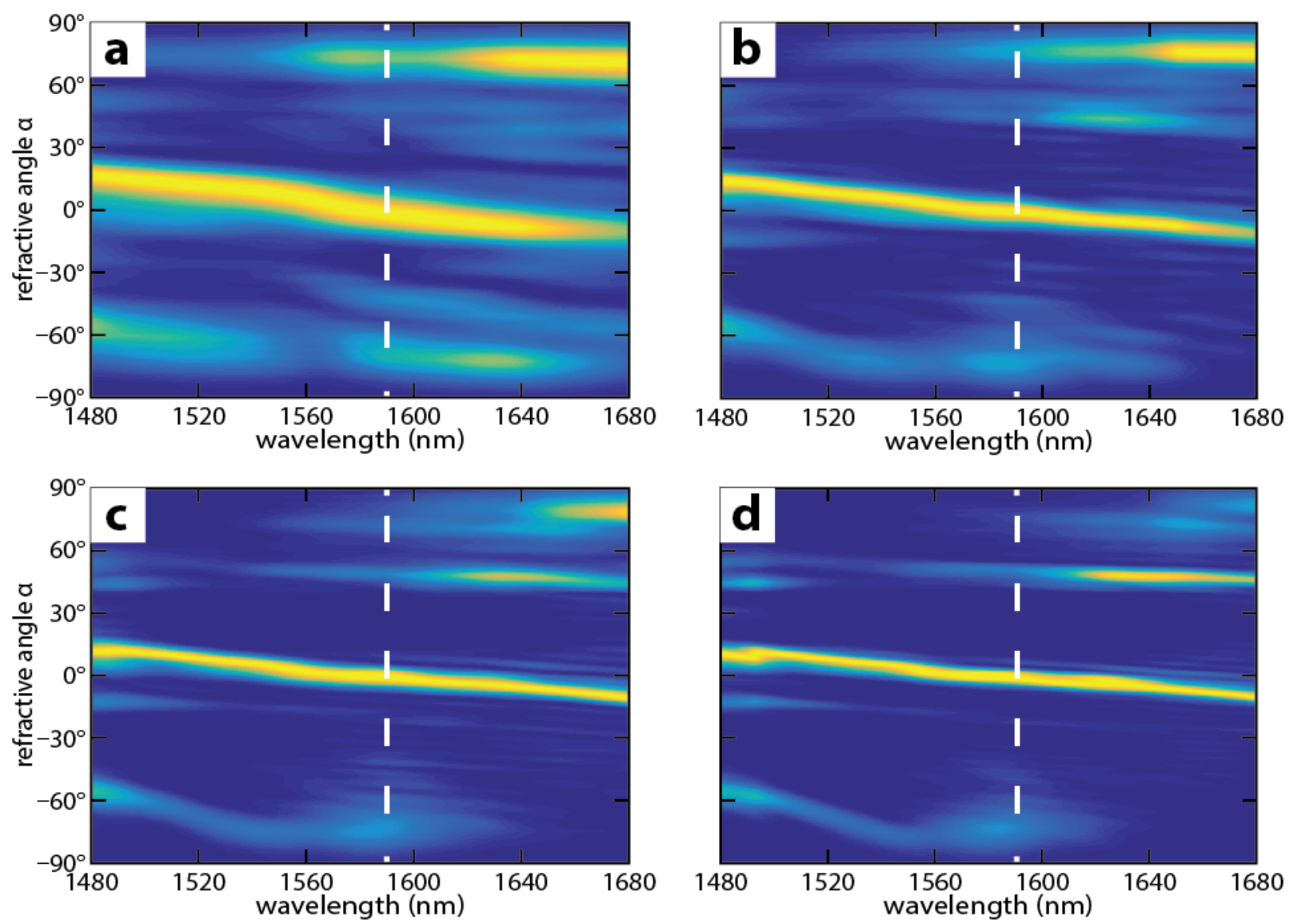

Fig.S1. Simulated far-field patterns for metamaterial prisms with (a) $5 \times 5$ pitches, (b) $10 \times 10$ pitches, (c) $15 \times 15$ pitches, and (d) $20 \times 20$ pitches. The white dashed line indicates the wavelength, $1590 \mathrm{~nm}$, at which the metamaterial is designed to exhibit a zero index (Section S.3). 


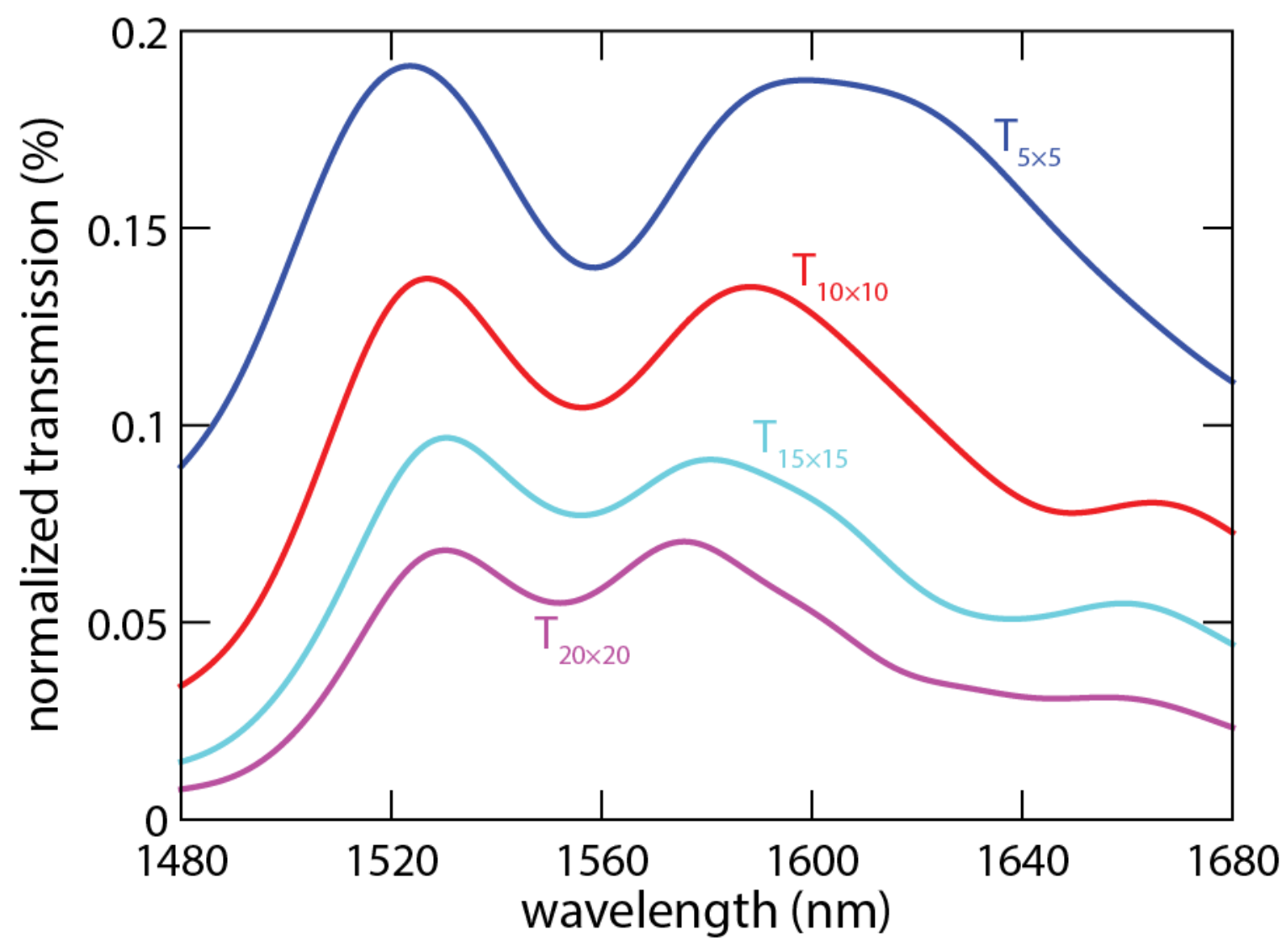

Fig.S2. Transmission of metamaterial prisms of various sizes: $5 \times 5$ pitches, $10 \times 10$ pitches, $15 \times 15$ pitches, and $20 \times 20$ pitches. This represents the total power transmitted from the output facet of the prism to the SU-8 slab waveguide (Fig. 3b). 

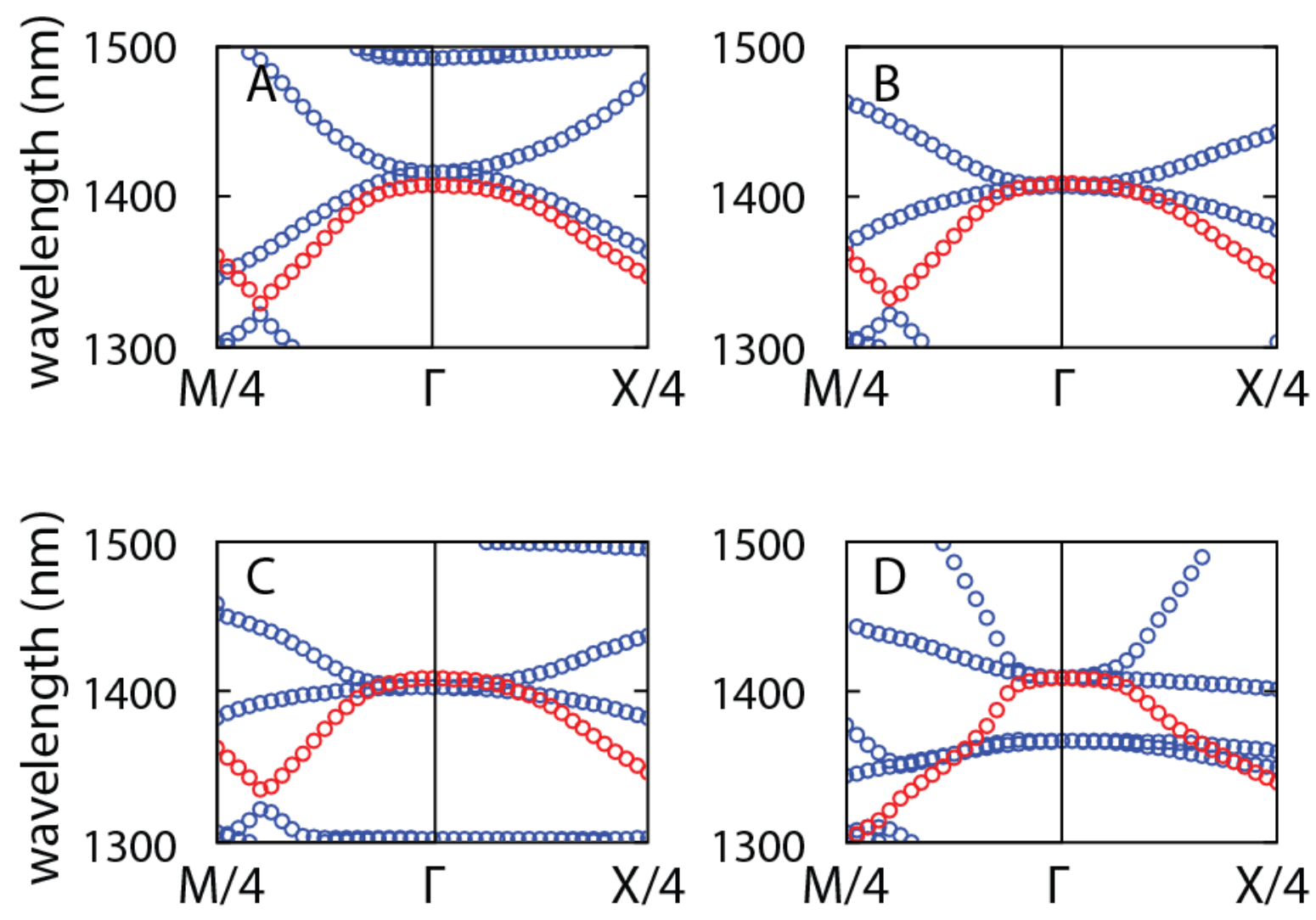

Fig.S3. Dirac cones correspond to the structures without the gold mirrors. Band structures corresponding to four different geometries that exhibit degeneracies for the electric monopole 
mode (red dots) and the transverse magnetic dipole mode. A, B, C and D correspond to SU-8 thicknesses of $687.5 \mathrm{~nm}, 1125 \mathrm{~nm}, 1575 \mathrm{~nm}$ and $1725 \mathrm{~nm}$, respectively.

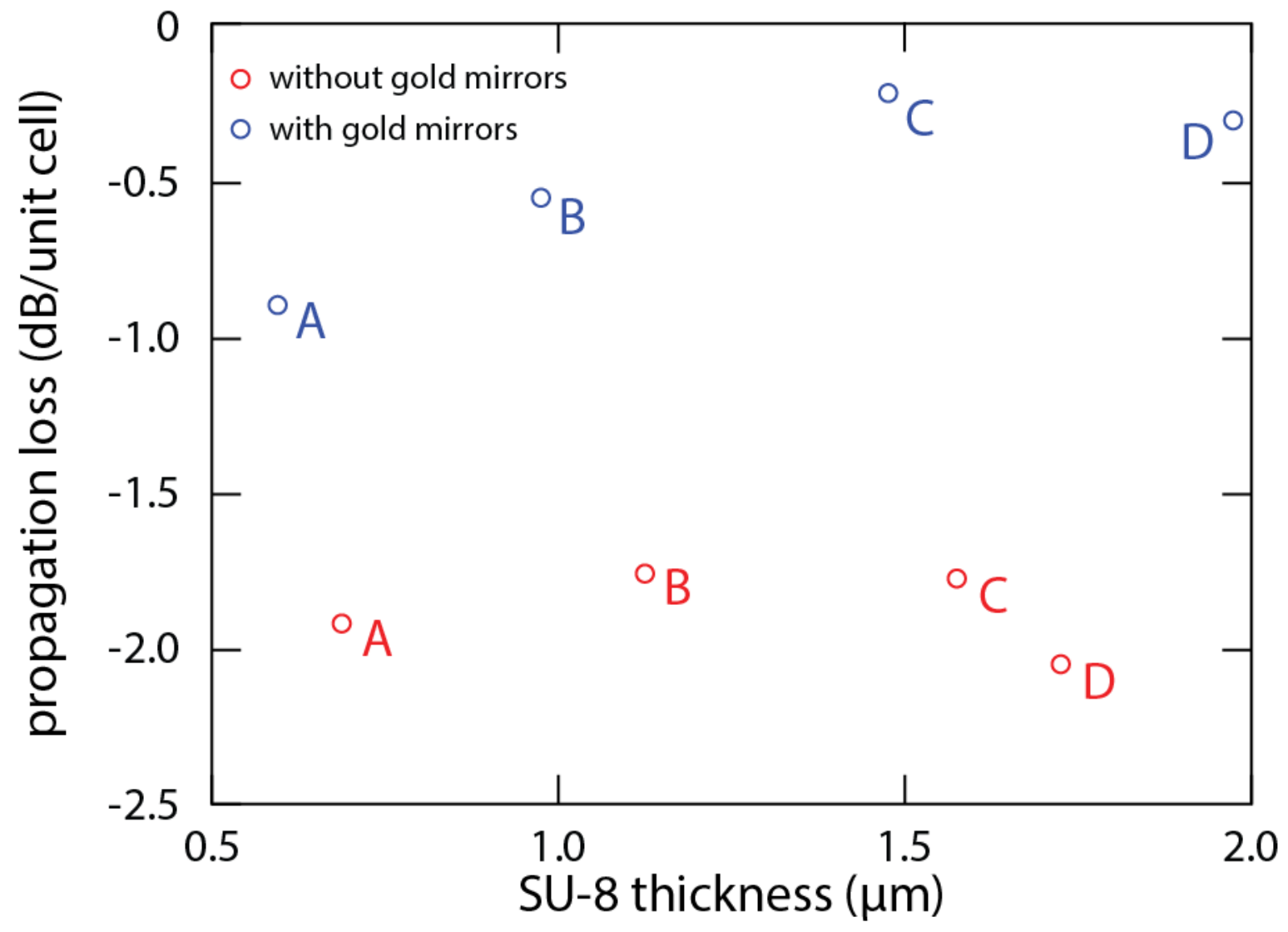

Fig.S4. Propagation loss for structures with modal degeneracies with (blue) and without (red) gold mirrors for the geometries (A, B, C, D) indicated in Fig. S3 and Fig. S15. 


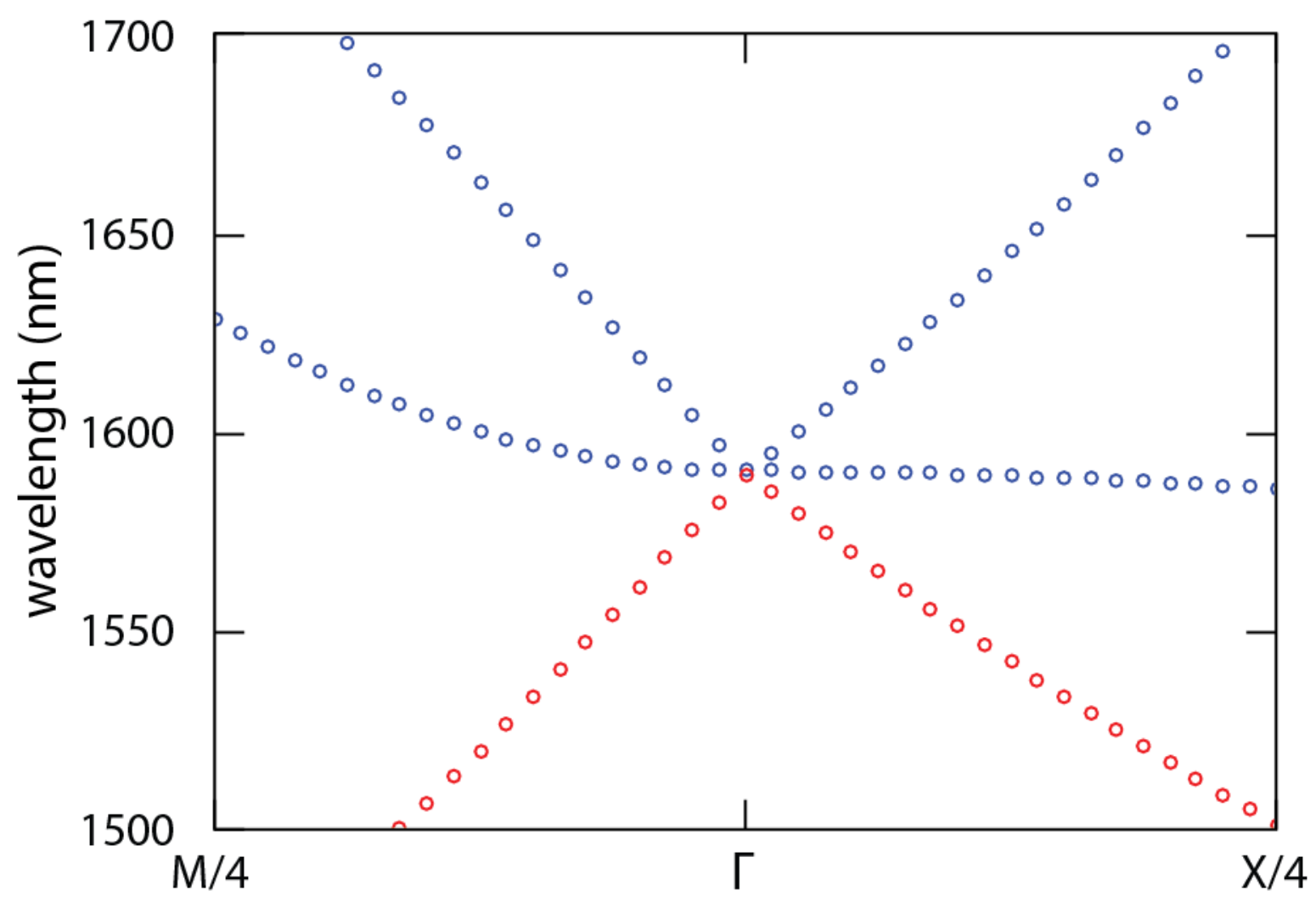

Fig.S5. Dirac cone corresponds to the structure without the gold caps on top of the silicon pillars for a radius of $189 \mathrm{~nm}$ and a pitch of $727 \mathrm{~nm}$. Red dots: electric monopole mode. This dispersion was designed using the method outlined in section S.3. 

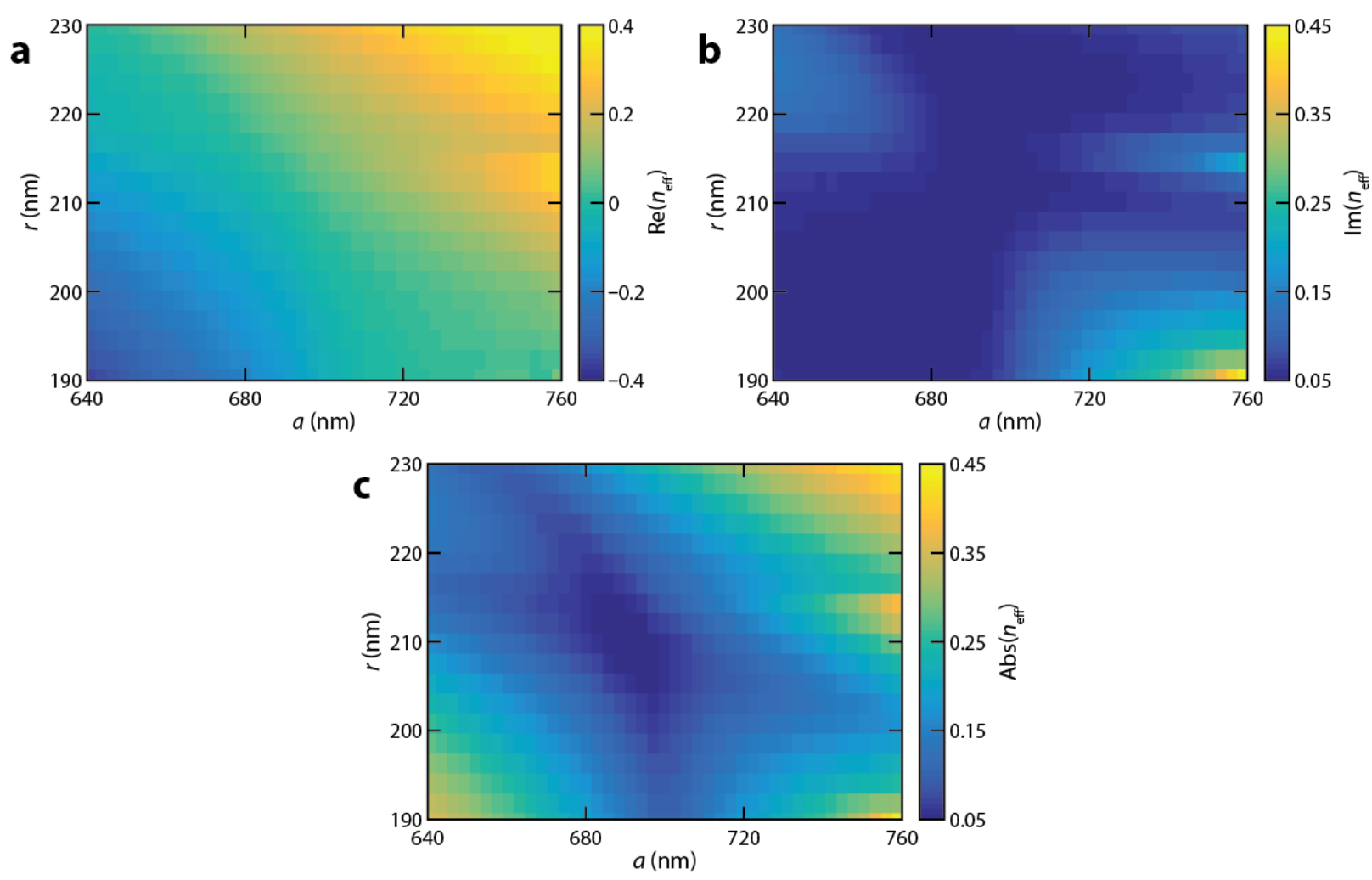

Fig.S6. Effective index $n_{\text {eff }}$ of the presented Dirac-cone metamaterial as a function of pitch $a$ and radius $r$ (Fig. 1a) at the design wavelength, $\lambda=1590 \mathrm{~nm}$ : (a) Real $n_{\text {eff }}$; (b) Imaginary $n_{\text {eff }}$; (c) Absolute value of $n_{\text {eff }}$. 


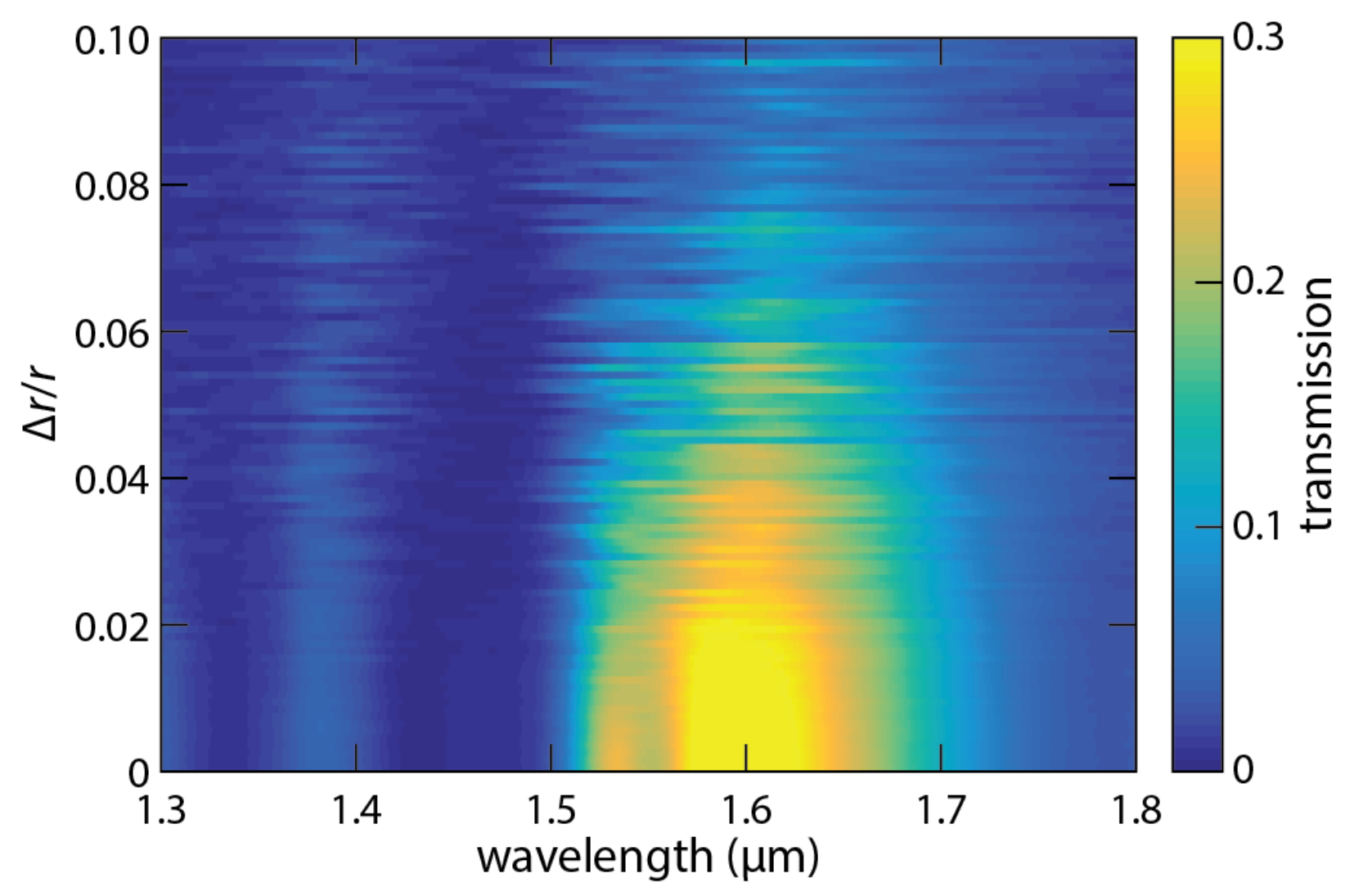

Fig.S7. Effect of disorder in pillar radius on the transmission through 8 unit cells. 


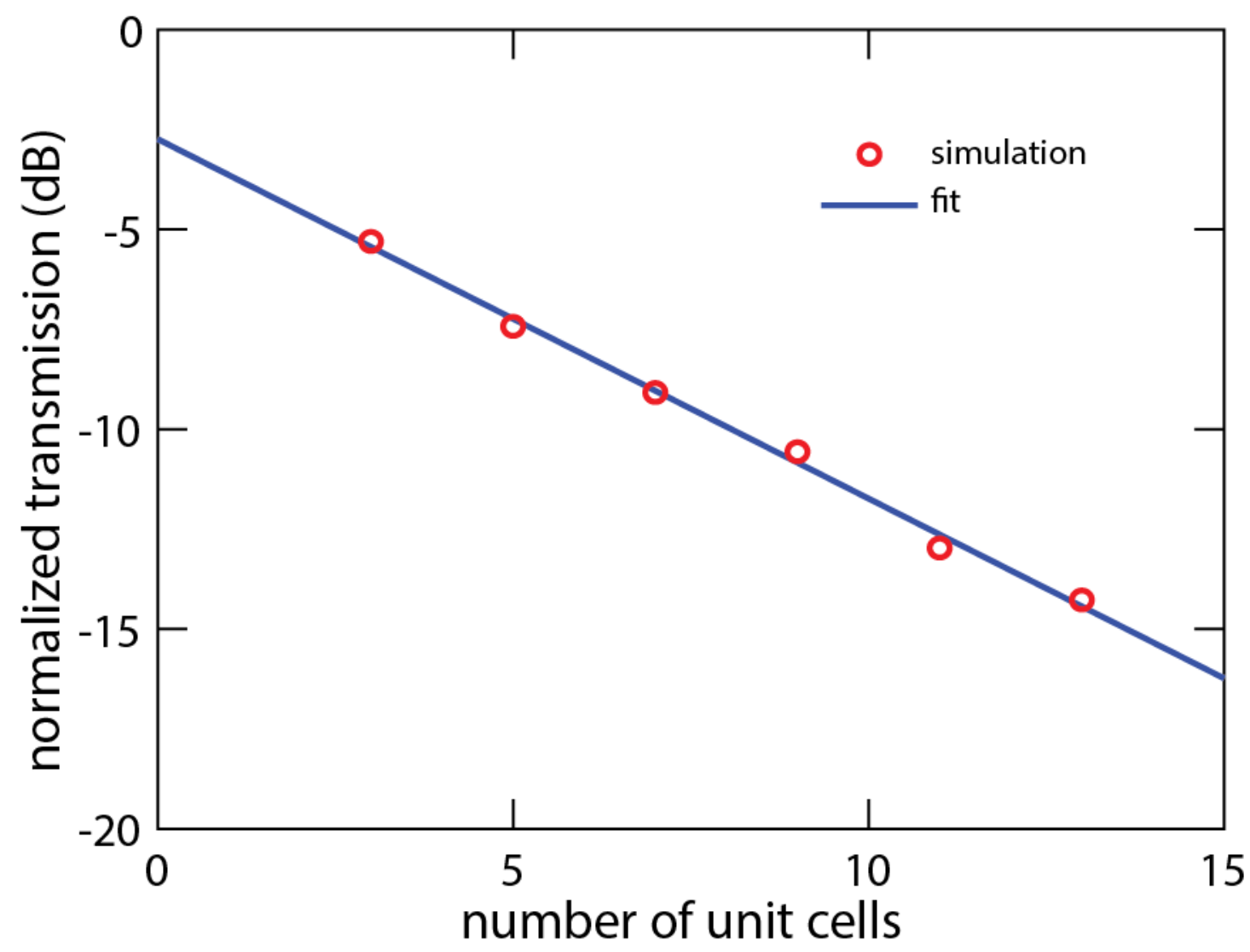

Fig.S8. Propagation loss as a function of number of unit cells: the slope of the line corresponds to the propagation loss; the intercept between the line and the $y$ axis corresponds to the coupling loss associated with the metamaterial. 


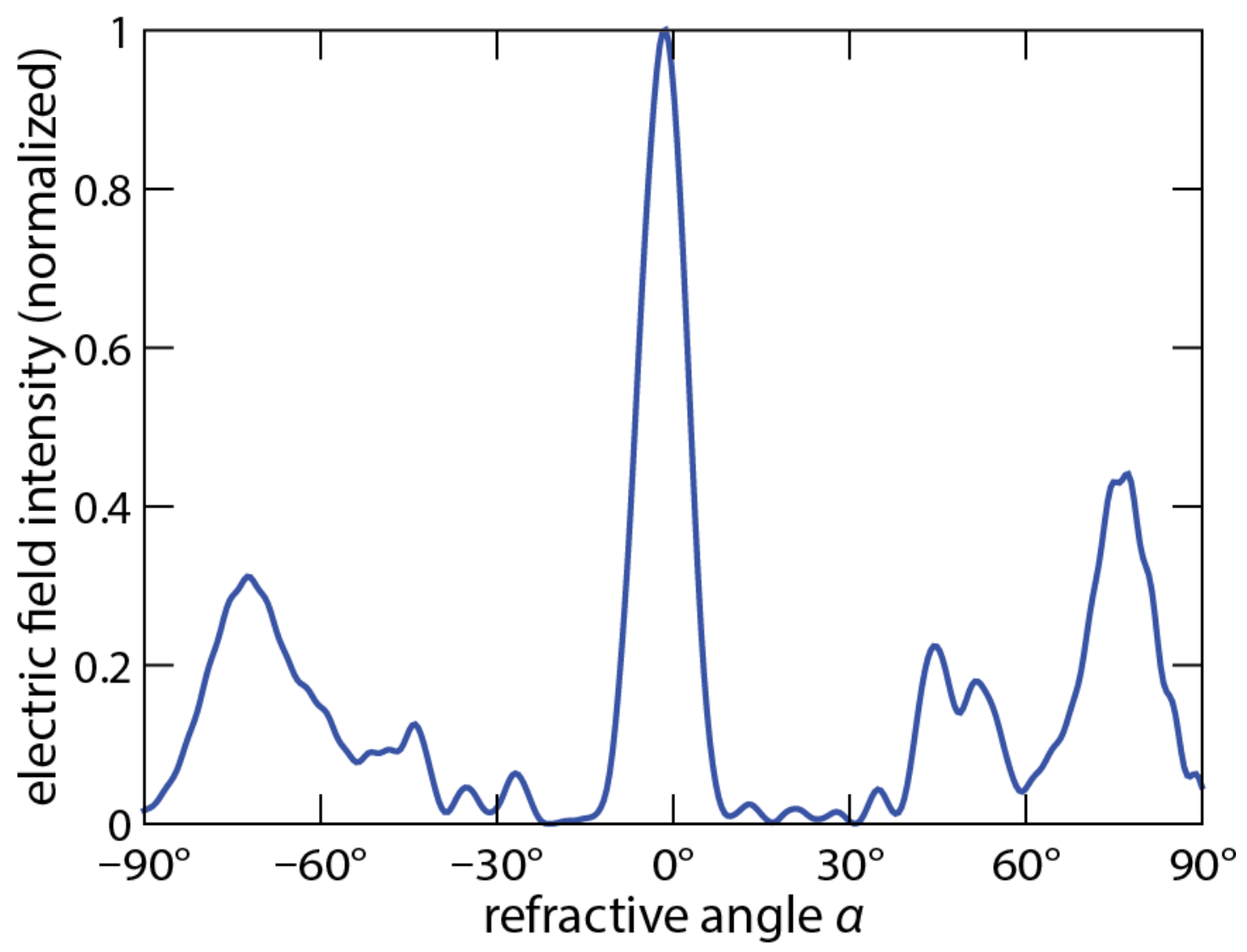

Fig.S9. Simulated far-field pattern for metamaterial prism with $10 \times 10$ pitches for the wavelength at which the metamaterial exhibits a designed zero index, $\lambda=1590 \mathrm{~nm}$ (Section S.3). 


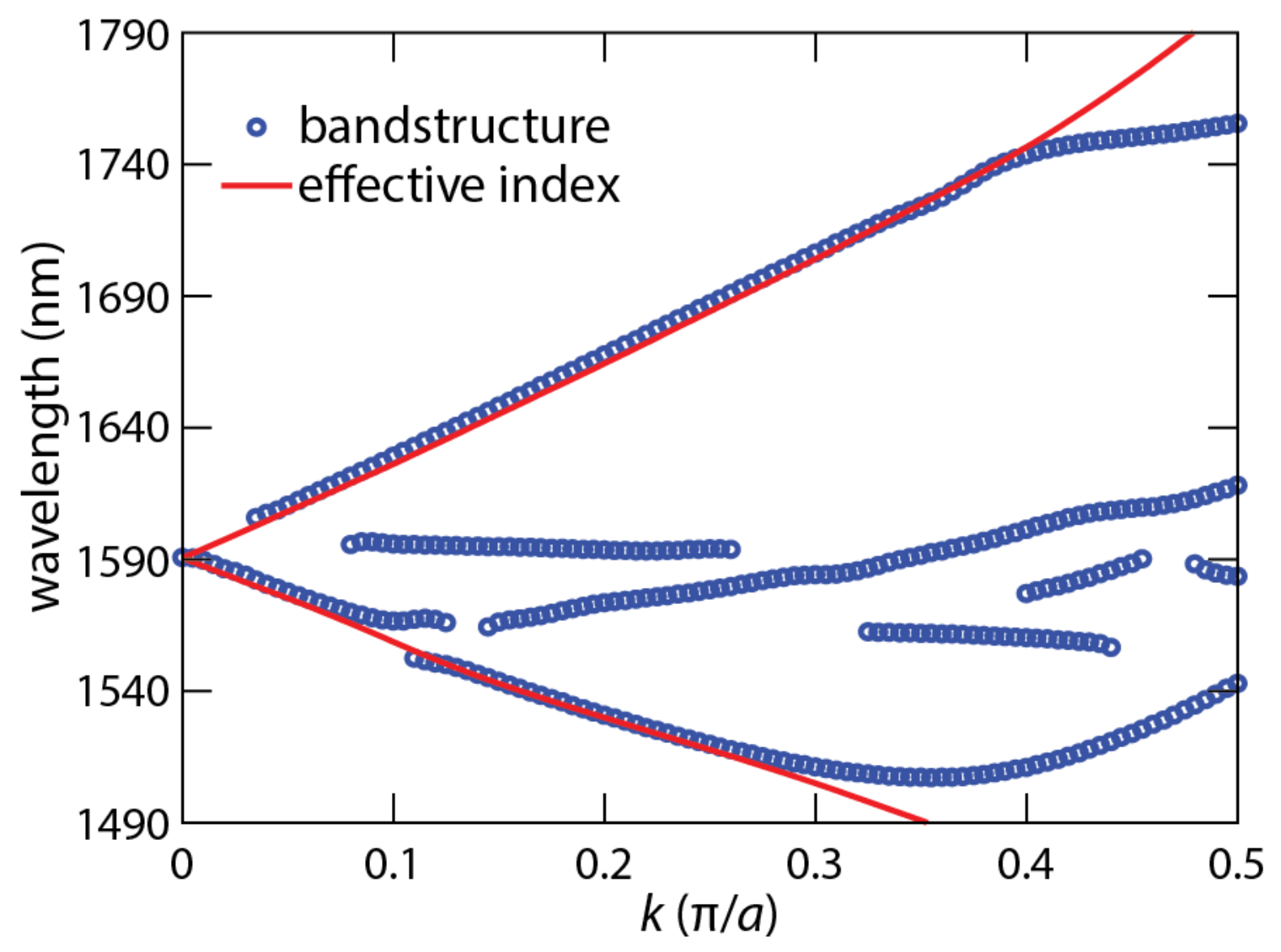

Fig.S10. Comparison of band structures of the presented metamaterial (Fig. 1a) computed by determining the angular frequencies as a function of wave vector for all the Bloch modes (blue dot), and by $k=n_{\text {eff }} \omega / c$ with the retrieved effective index $n_{\text {eff }}$ (red curve). 
a

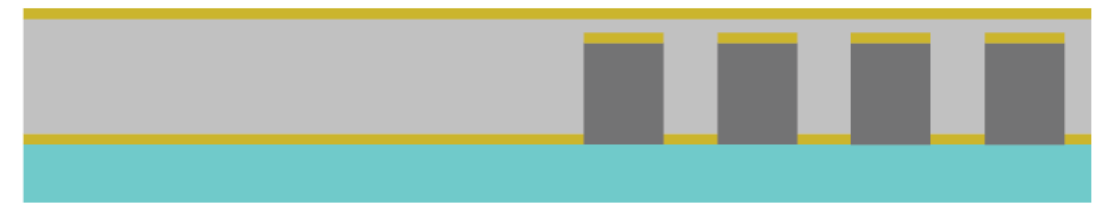

\section{bulk medium}
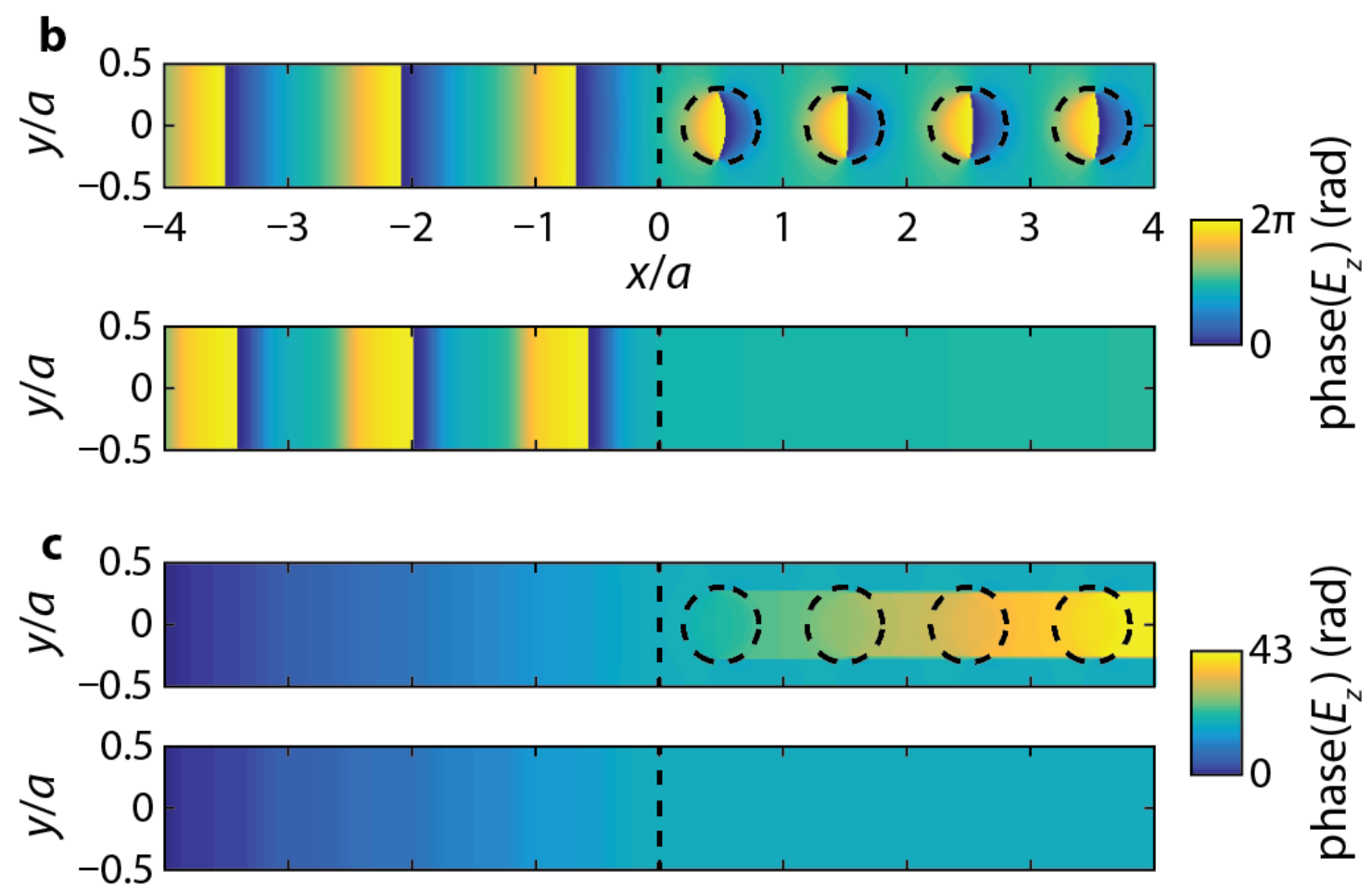

d

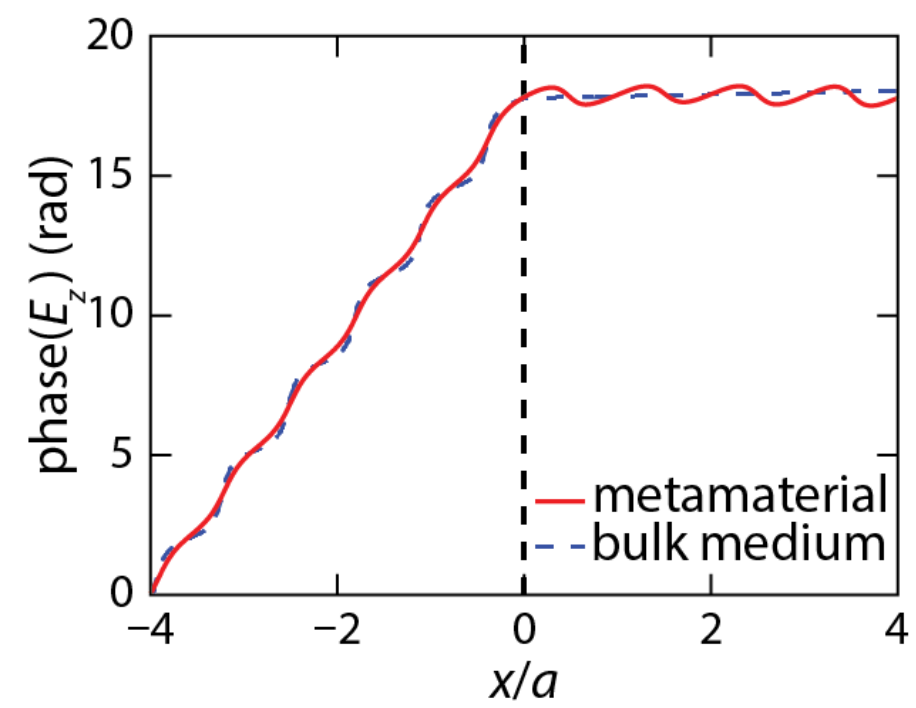


Fig.S11. The phase of the electric field within a Dirac-cone metamaterial compared with that of a homogenized zero-index medium near the interface with an input waveguide. (a): Cross-sectional views of the Dirac-cone metamaterial (top, parameters are as in Fig. 1a) and its homogenized model (bottom). The input waveguide consists of SU-8 clad by gold films, and the homogenized zero-index medium is defined by the retrieved constitutive parameters at $1590 \mathrm{~nm}$ in Fig. 2e. (b): Phase of $E_{\mathrm{z}}$ in the range of $[0,2 \pi]$ of the Dirac-cone metamaterial (top) and its homogenized model (bottom) in a plane parallel to the substrate. The phase corresponds to the electric field perpendicular to the plane of propagation, $E_{z}$ at a wavelength of $1590 \mathrm{~nm}$. The dashed lines indicate the interface (the left edge of the metamaterial unit-cell or the boundary of the homogenized model) and pillar positions within the Dirac-cone metamaterial. (c): Same phase as in (b) but unwrapped to show constant phase advance in the SU-8 region. (d): Comparison of the unwrapped phase along the propagation direction in the Dirac-cone metamaterial and the 
corresponding homogenized model. The phase is sampled along the top or bottom edge of the simulation region in (c).
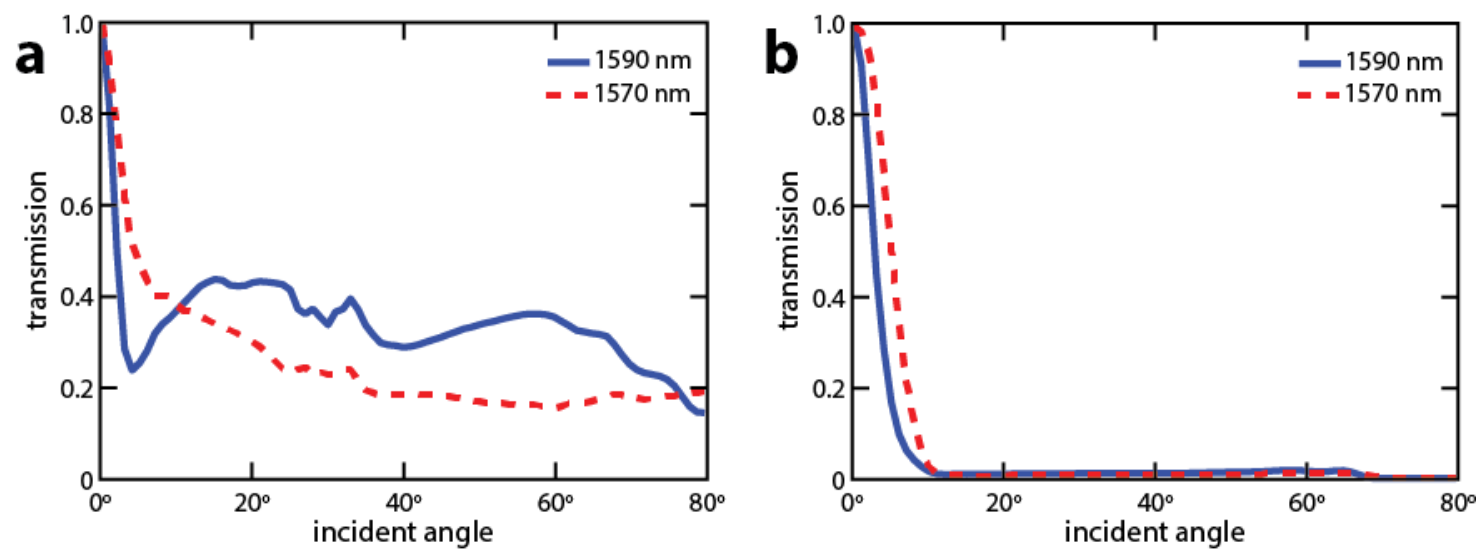

Fig.S12. Angle-dependent transmission of (a) the Dirac-cone metamaterial and (b) its homogenized model. Transmission is normalized to its maximum value.

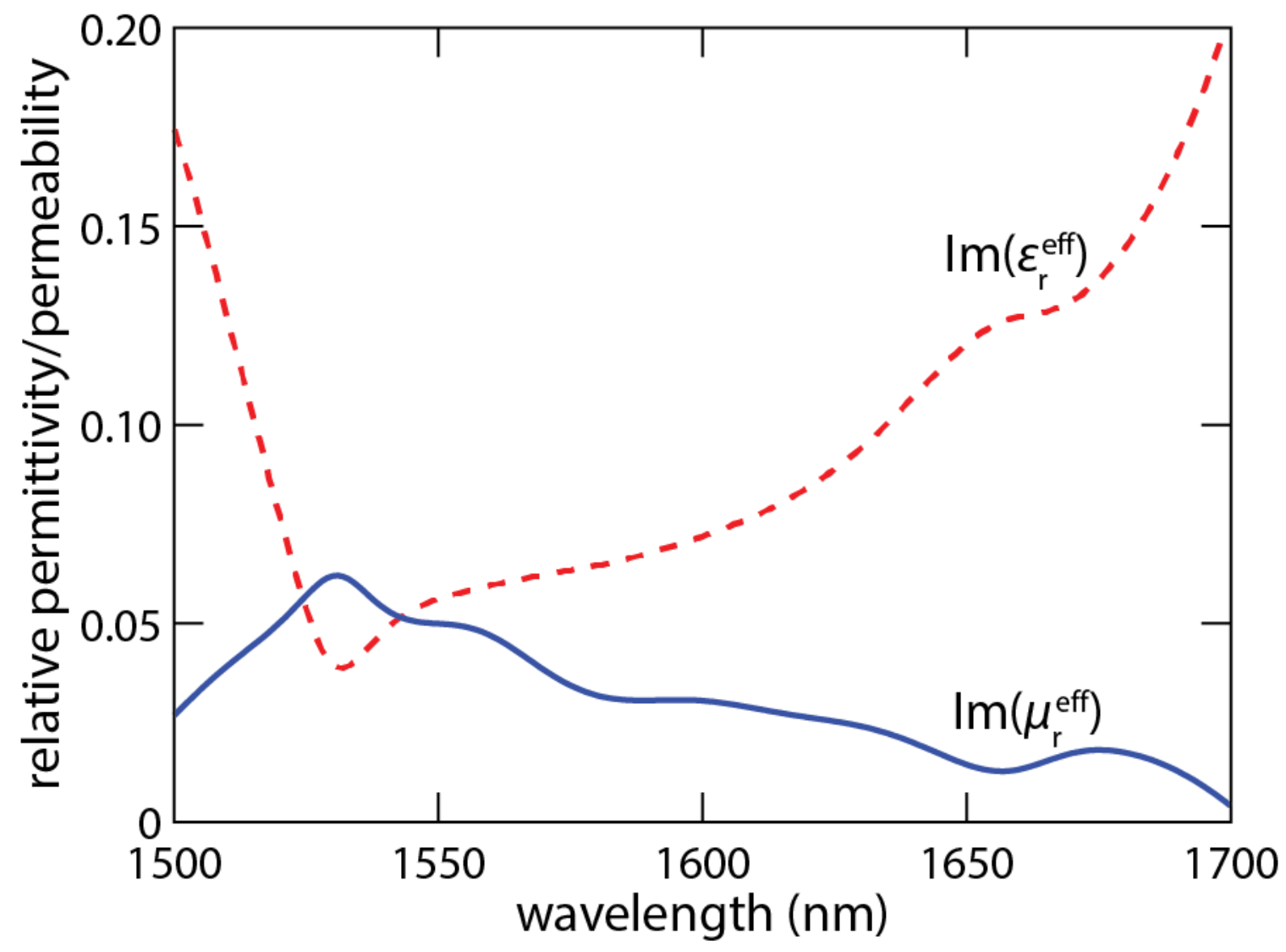


Fig.S13. Imaginary parts of the effective relative permittivity and permeability of the metamaterial retrieved from numerically calculated reflection and transmission coefficients.

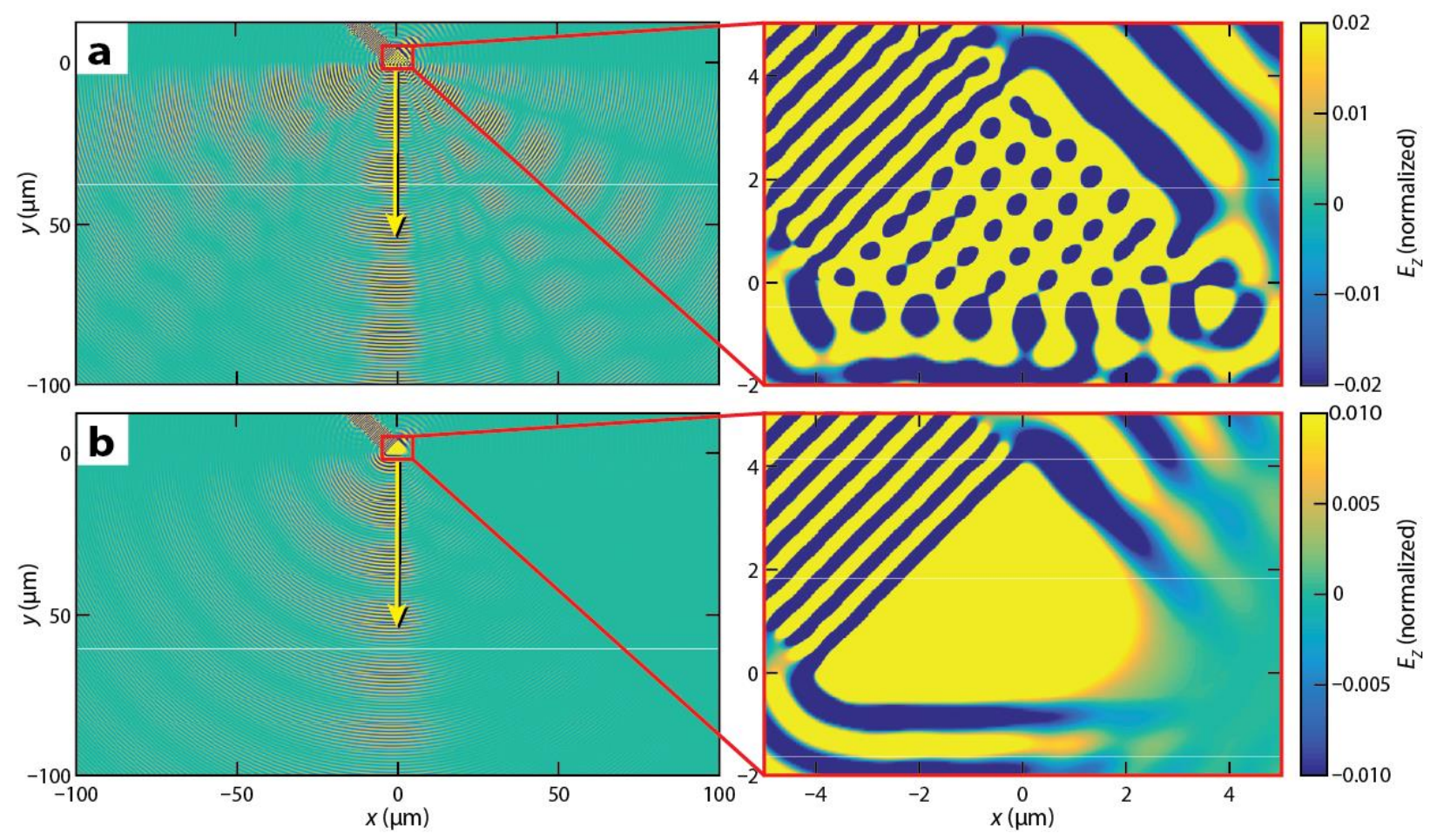

Fig.S14. Simulated $E_{z}$ in the prism and SU-8 slab waveguide region at $1590 \mathrm{~nm}$. Comparison of (a): a metamaterial prism (parameters are as in Fig. 1a), and (b): a prism composed of a homogenized model based on the retrieved constitutive parameters from Fig. 2e. 


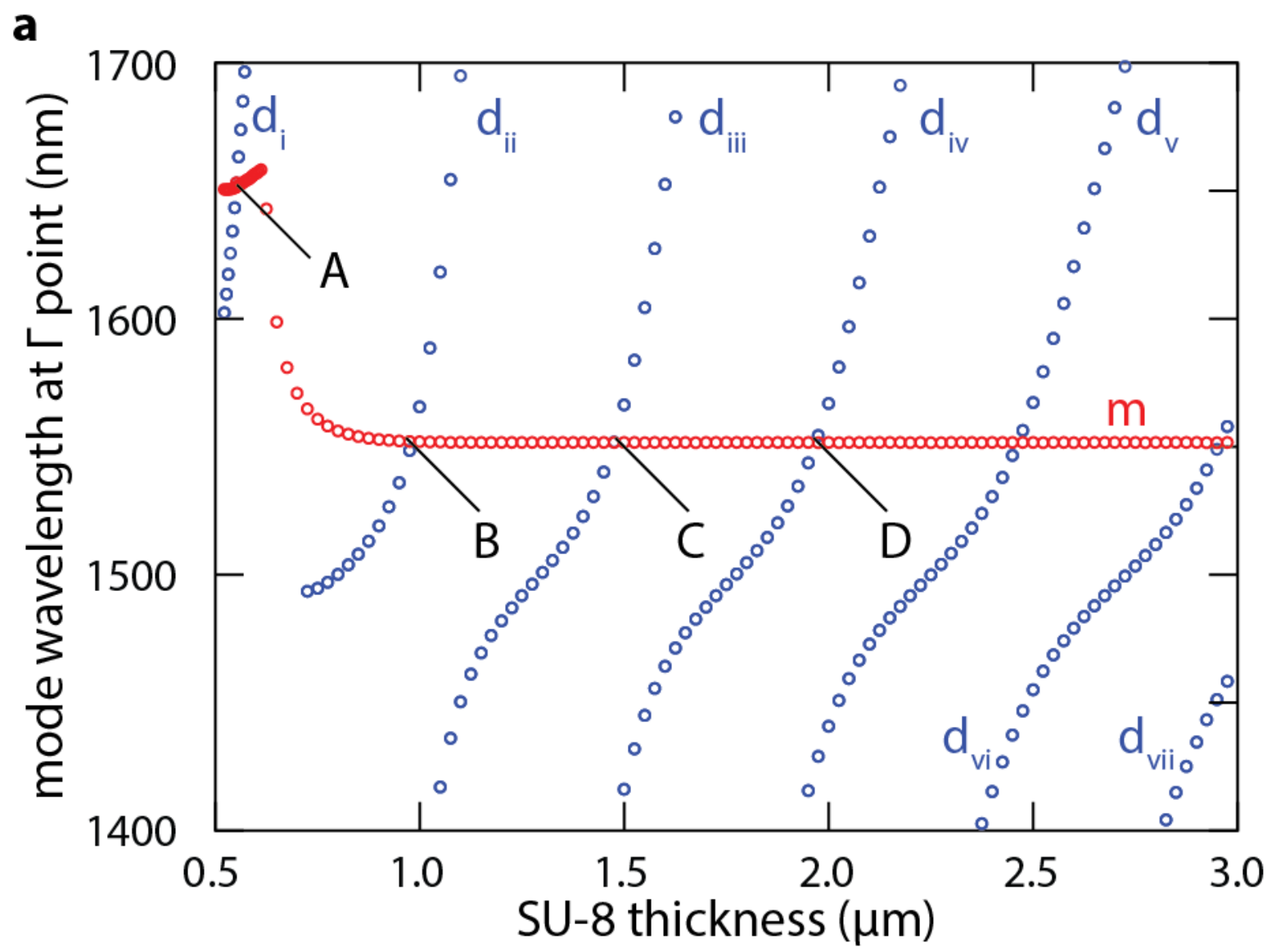

b
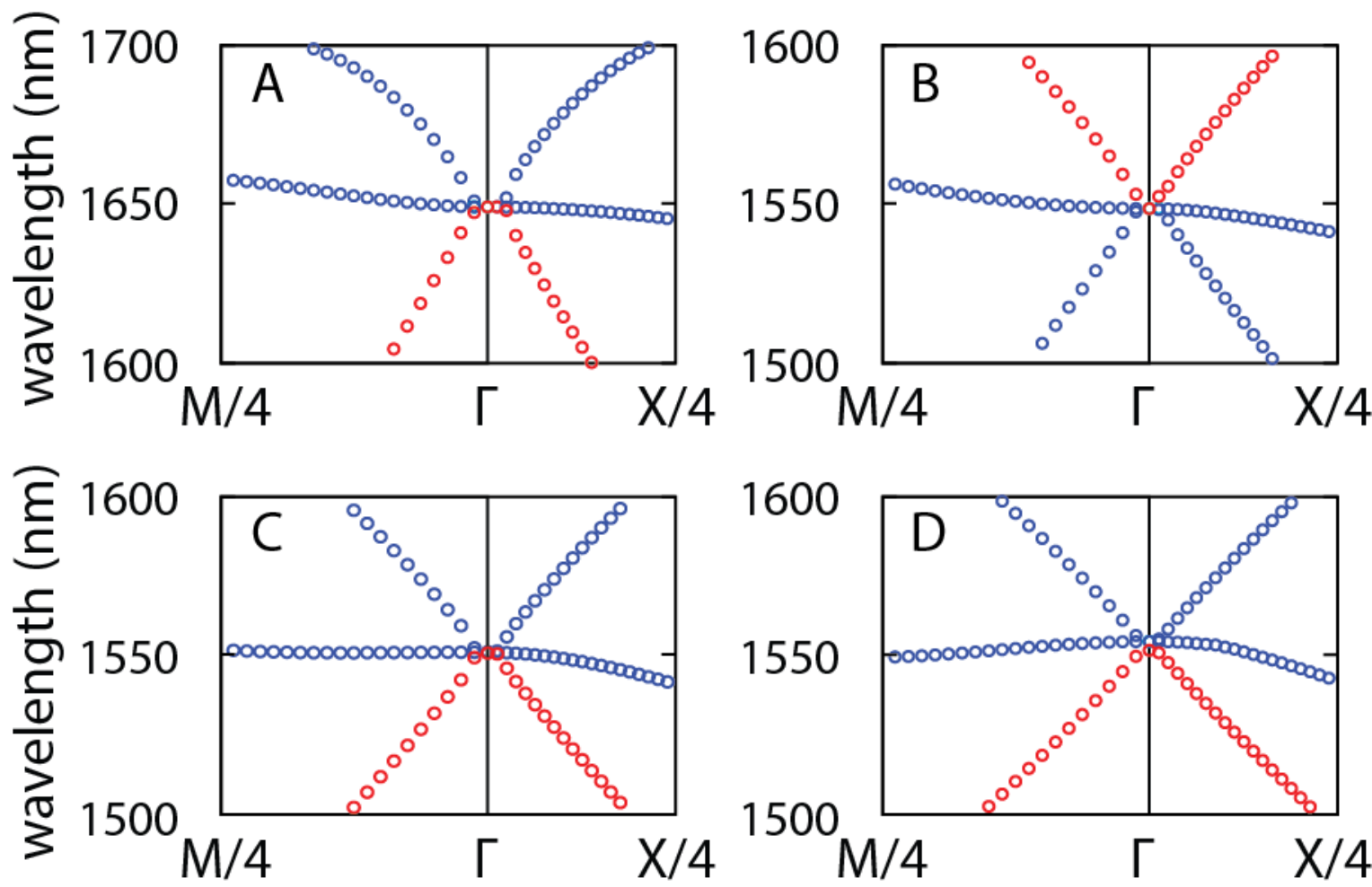
Fig.S15. Effect of variation in SU-8 thickness $h_{\mathrm{SU}-8}$ on modes forming the Dirac cone. (a): Wavelengths of the electric monopole mode (m: red curve) and the transverse magnetic dipole modes $\left(\mathrm{d}_{\mathrm{i}}-\mathrm{d}_{\mathrm{vii}}\right.$ : blue curve) at $\Gamma$ point for various $h_{\mathrm{SU}-8}$. (b): Band structures corresponding to 
the four intersections (A, B, C, D) of the electric monopole mode and the four different transverse magnetic dipole modes $\left(\mathrm{d}_{\mathrm{i}}-\mathrm{d}_{\mathrm{iv}}\right.$ in $\left.(\mathbf{a})\right)$.
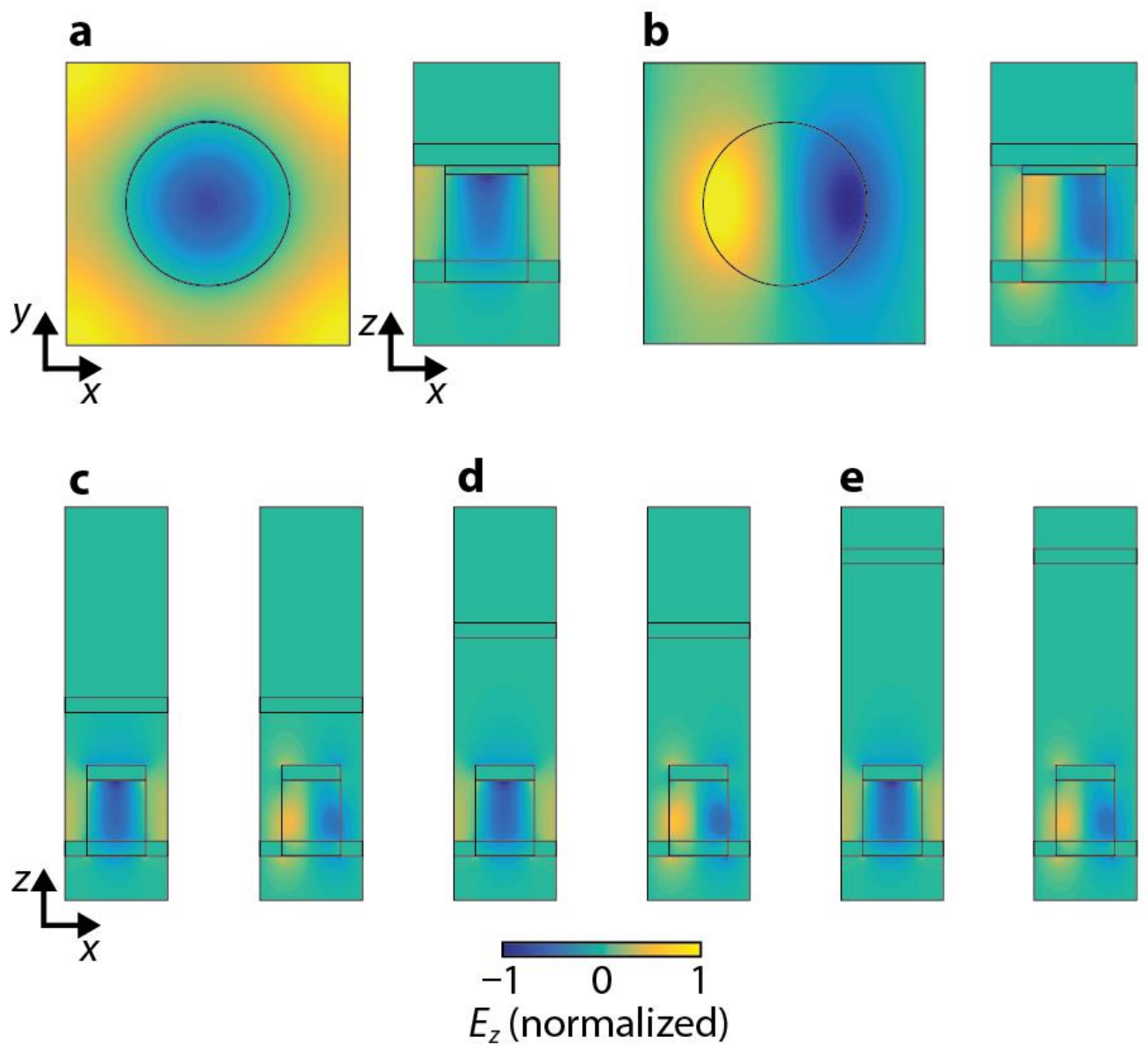

Fig.S16. Simulated mode profiles in one unit-cell of metamaterials with different SU-8 thicknesses $h_{\mathrm{SU}-8}$ at the Dirac-point wavelength (Fig. S15b). (a) $x y$-plane and $x z$-plane $E_{\mathrm{z}}$ corresponding to the electric monopole mode of the metamaterial with $h_{\mathrm{SU}-8}=550 \mathrm{~nm}$. (b) $x y$-plane and $x z$-plane $E_{\mathrm{z}}$ corresponding to the transverse magnetic dipole mode of the metamaterial with $h_{\mathrm{SU}-8}=550 \mathrm{~nm}$ 
. (c-e) $x z$-plane $E_{z}$ corresponding to the electric monopole mode (left) and transverse magnetic dipole mode (right) of the metamaterial with $h_{\mathrm{SU}-8}=975 \mathrm{~nm}, 1475 \mathrm{~nm}$ and $1975 \mathrm{~nm}$, respectively.
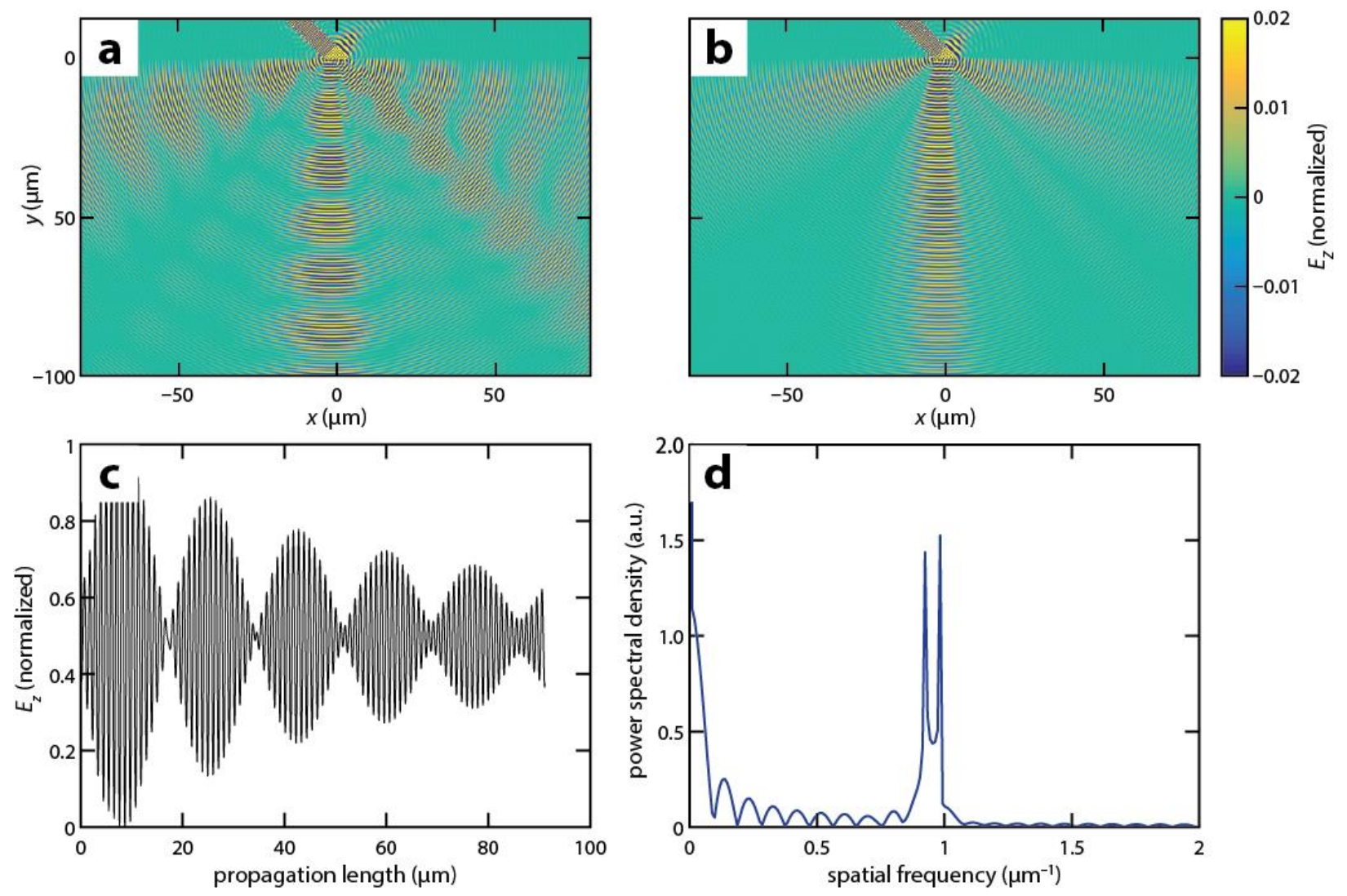

Fig.S17. Simulated $E_{\mathrm{z}}$ and interference patterns in the spatial and spectral domains. Comparison of the (a) simulated $E_{z}$ for our fabricated prism structure when coupled to a $2-\mu \mathrm{m}$-thick SU-8 slab waveguide and (b) our fabricated prism structure when coupled to a $0.8-\mu \mathrm{m}$-thick SU-8 slab 
waveguide. (c) $E_{\mathrm{z}}$ sampled in a plane perpendicular to the propagation plane along the refracted beam and (d) its corresponding Fourier transform.
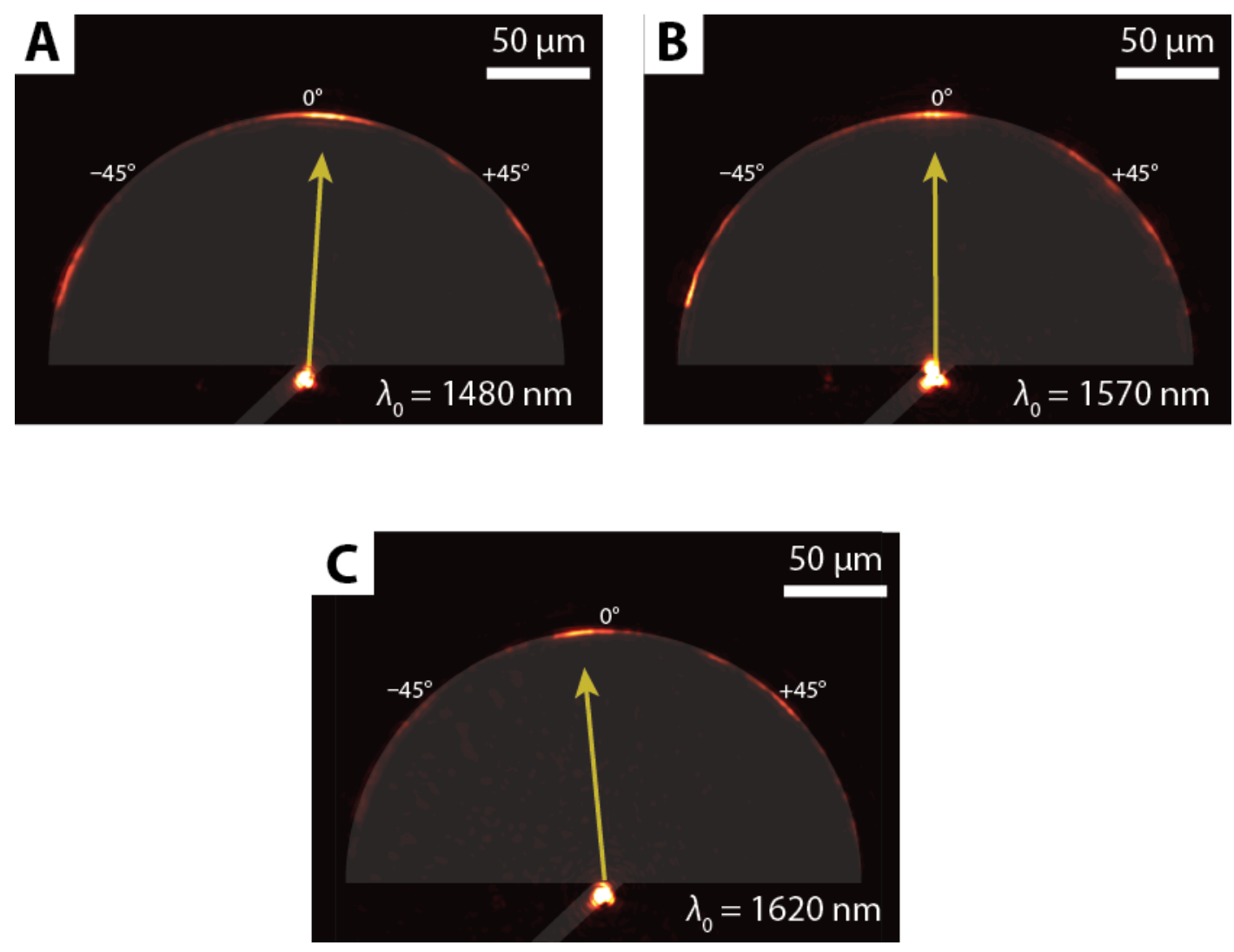

Fig.S18. Near-infrared microscope images of the prism and SU-8 slab waveguide region (Fig. 1b, c) at (a) $1480 \mathrm{~nm},(\mathbf{b}) 1570 \mathrm{~nm}$, and (c) $1620 \mathrm{~nm}$. 
a
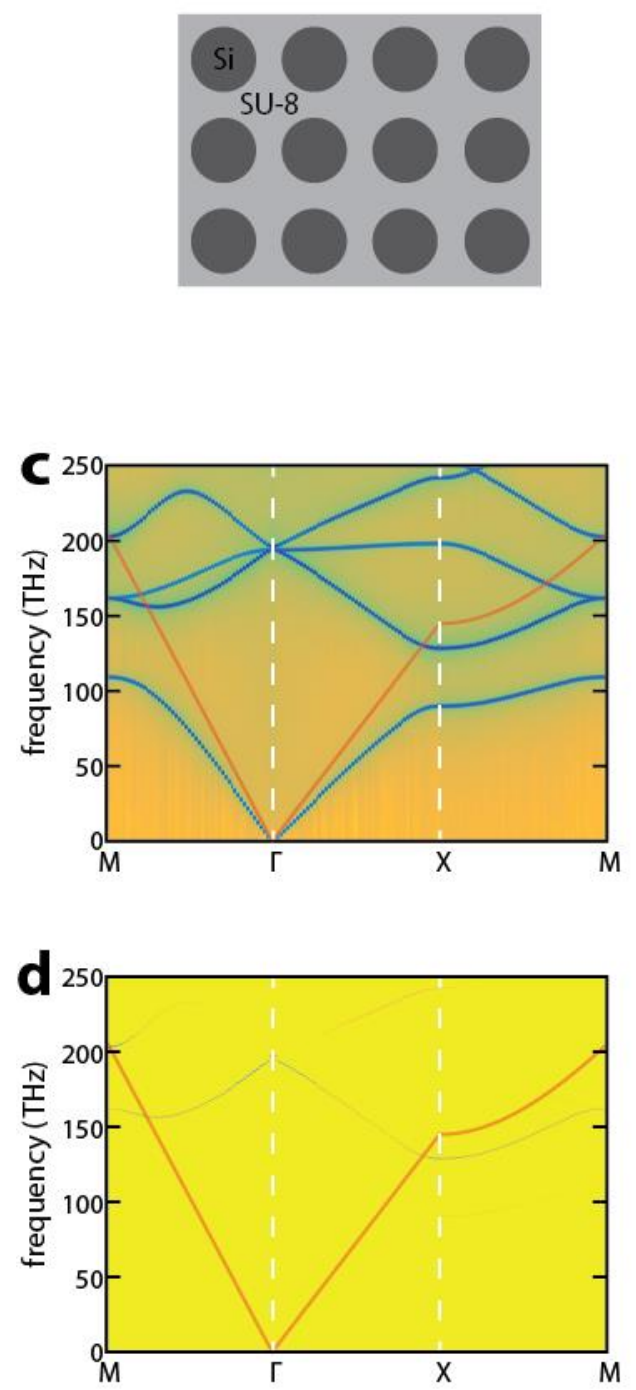
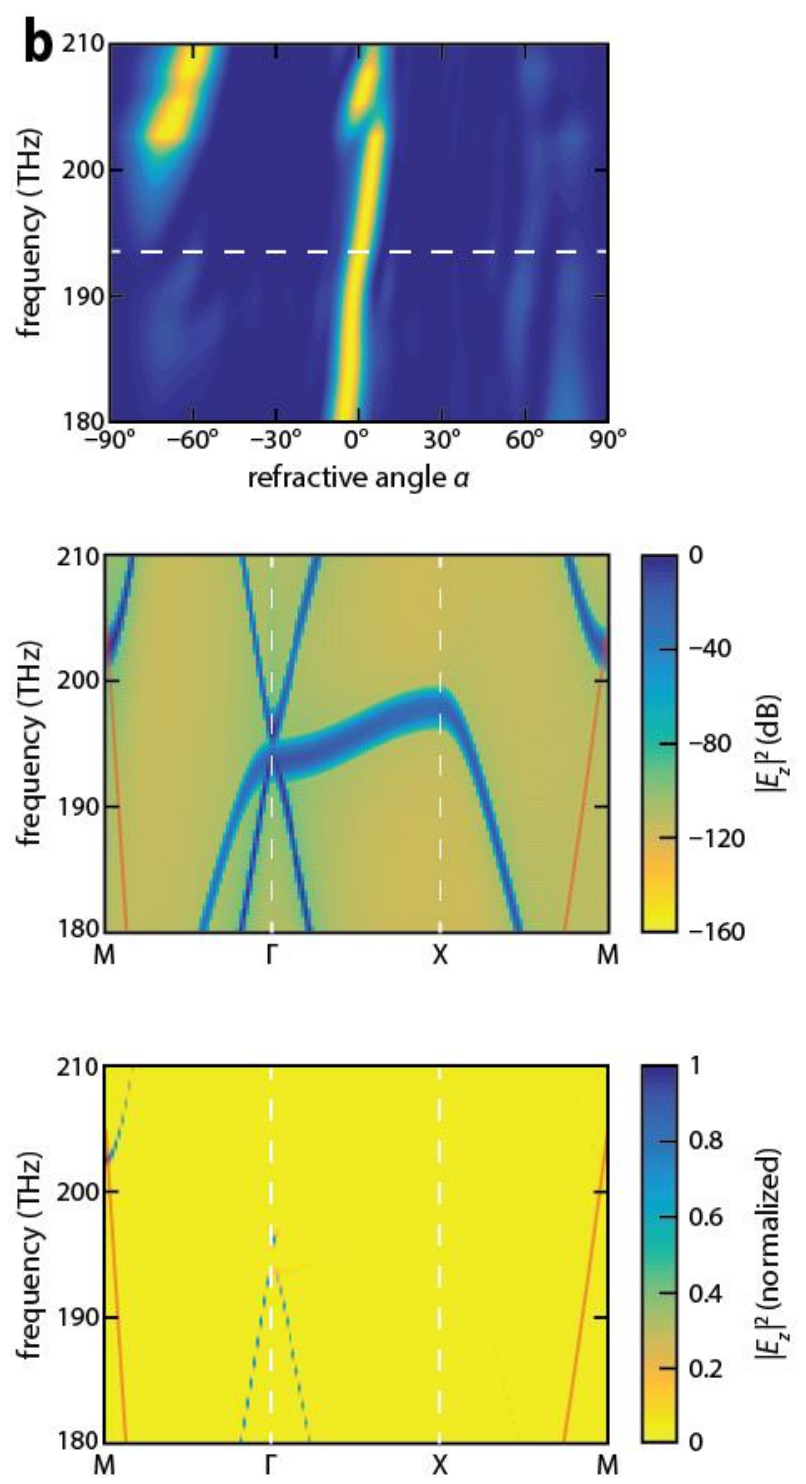

Fig.S19. 2D metamaterial with its corresponding band structure and far-field pattern. (a) The metamaterial consists of a 2D square array of infinitely long silicon pillars in an SU-8 matrix. The pitch and radius of the silicon pillars are $659.5 \mathrm{~nm}$ and $170.25 \mathrm{~nm}$, respectively. (b) Simulated farfield pattern of a metamaterial prism consisting of $8 \times 8$ pitches, whose output facet is coupled to a 2D semi-circular slab of SU-8 (cross-section is the same as that in Fig. 1b). The white dashed line indicates the frequency, $193.55 \mathrm{THz}(1550 \mathrm{~nm})$, at which the $2 \mathrm{D}$ metamaterial shows a zero 
index. (c) Photonic band structure of this 2D metamaterial for TM modes. The red lines are the light lines of the output 2D SU-8 slab waveguide. (d) Same as (c) but in linear scale.
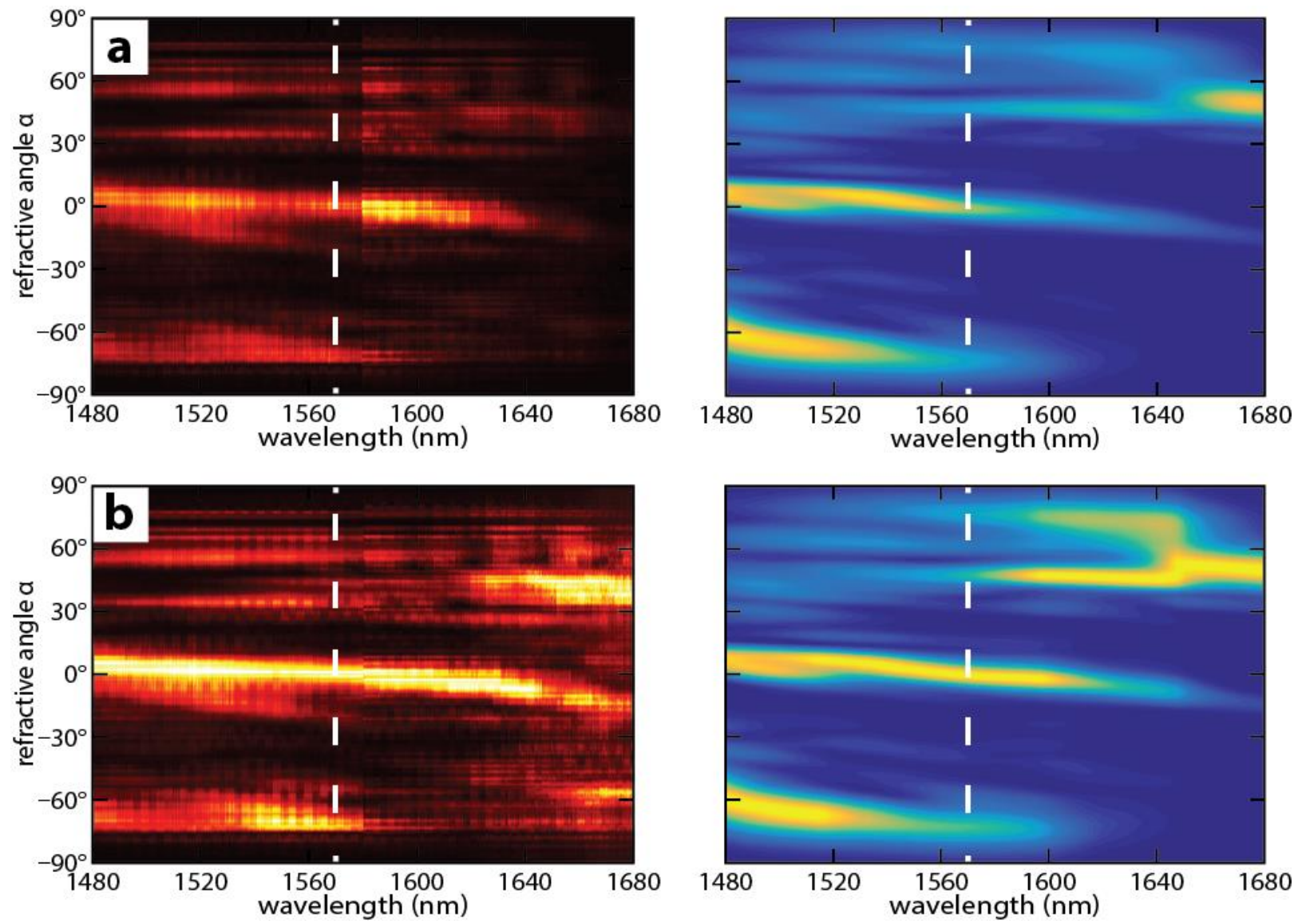

Fig.S20. Measured (left) and simulated (right) far-field patterns normalized to global maximum, (a), and at each wavelength, (b). The white dashed line indicates the wavelength, $1570 \mathrm{~nm}$, at which the refracted beam crosses $0^{\circ}$. 

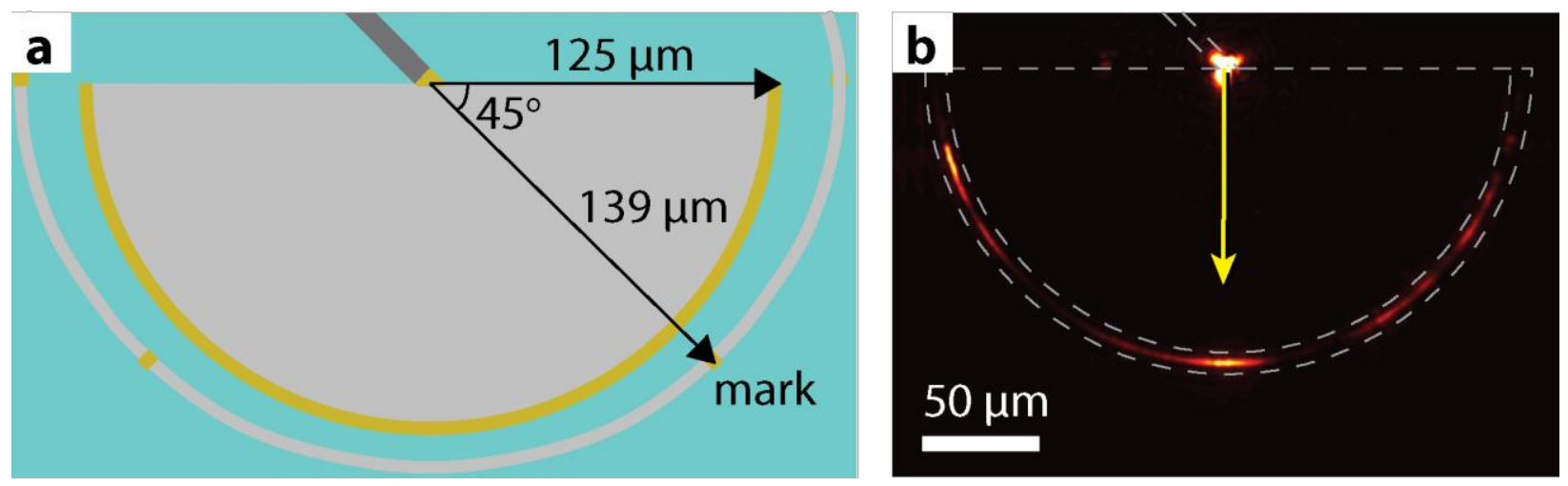

Fig.S21. Prism-based measurement configuration. (a) Schematic and (b) microscope image of the measurement configuration. In (a), a silicon waveguide directs light into a metamaterial prism, which couples to an SU-8 slab waveguide. A separate SU-8 waveguide, outside of the SU-8 slab waveguide, is used to illuminate alignment marks. In (b), white dashed lines indicate the position of the silicon waveguide and the SU-8 slab waveguide.
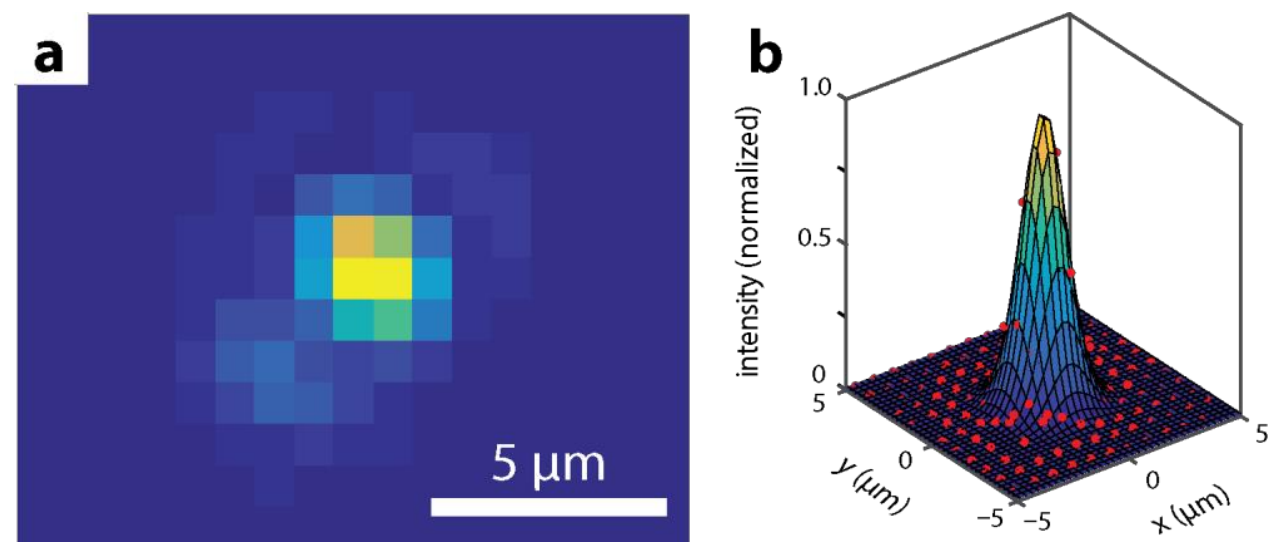

Fig.S22. Location of illuminated alignment marks. (a) InGaAs image of an alignment mark. (b) 2D Gaussian fit of the measured intensity. Red dots: measured intensities; colored surface: 
Gaussian surface fit. In (b), we fit the measured intensity values to a 2D Gaussian model using a nonlinear least-squares algorithm.
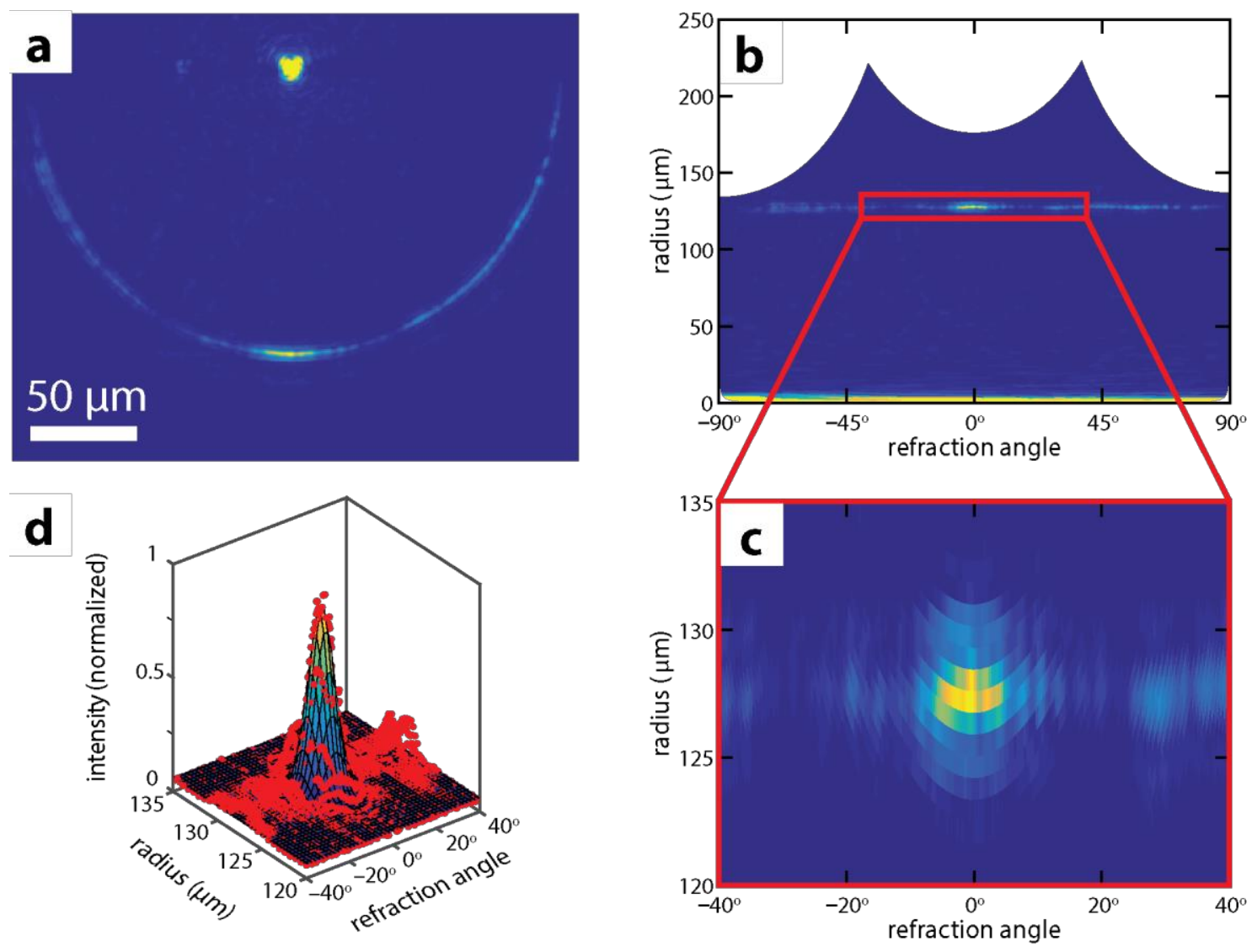

Fig.S23. Extracting refraction angle from InGaAs images. (a) Measured intensity values in the Cartesian coordinate. (b) Measured intensity values mapped to the polar coordinate. From (a) to (b), the curved edge of the SU-8 slab waveguide is transformed to a horizontal line. (c) Magnified view of the refracted beam region. (d) 2D Gaussian fit of the measured intensity in (c). Red dots: 
measured intensities; colored surface: Gaussian surface fit. In (d), we fit the measured intensity values to a 2D Gaussian model to extract the refracted angle.

a

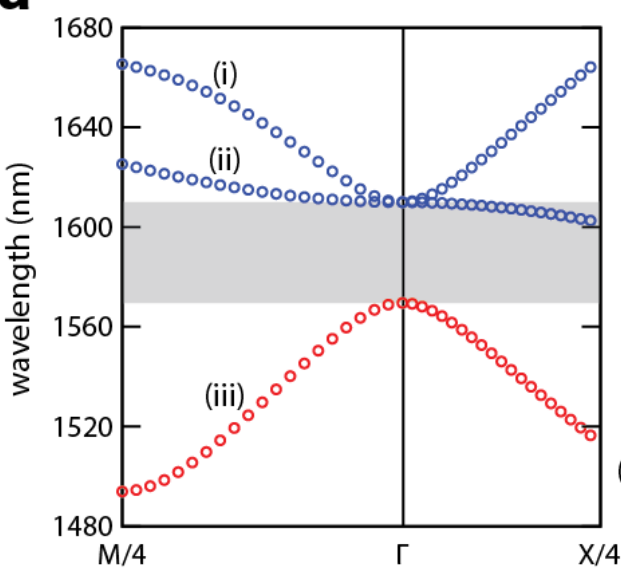

C

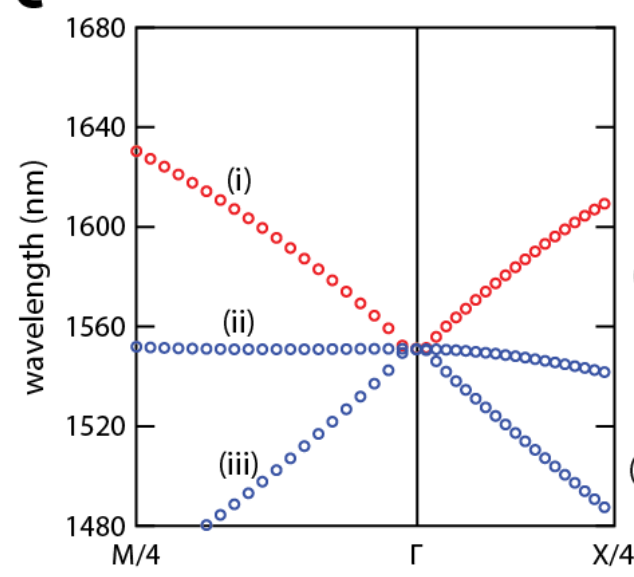

(i)

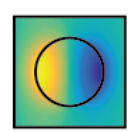

(ii)

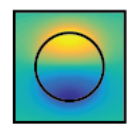

(iii)

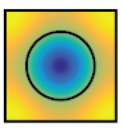

b

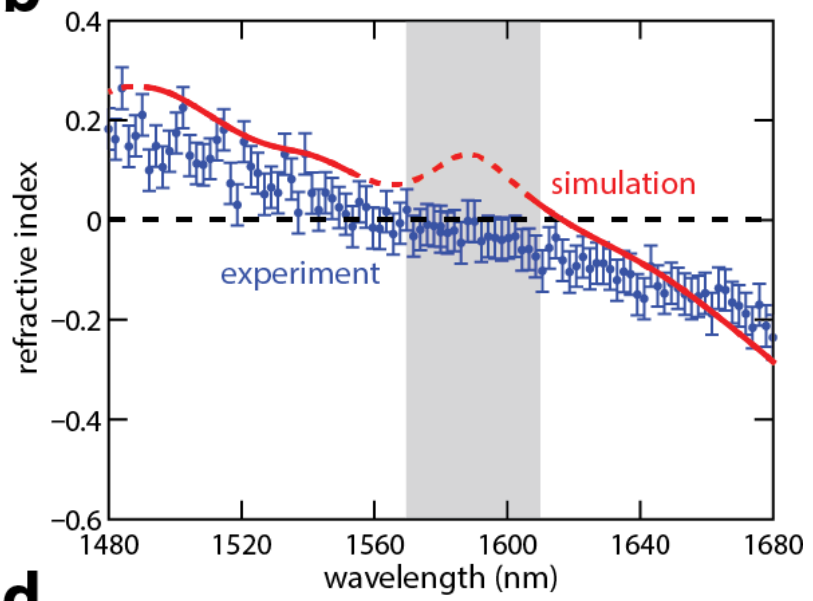

(i)

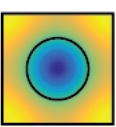

(ii)

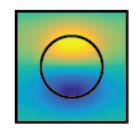

d

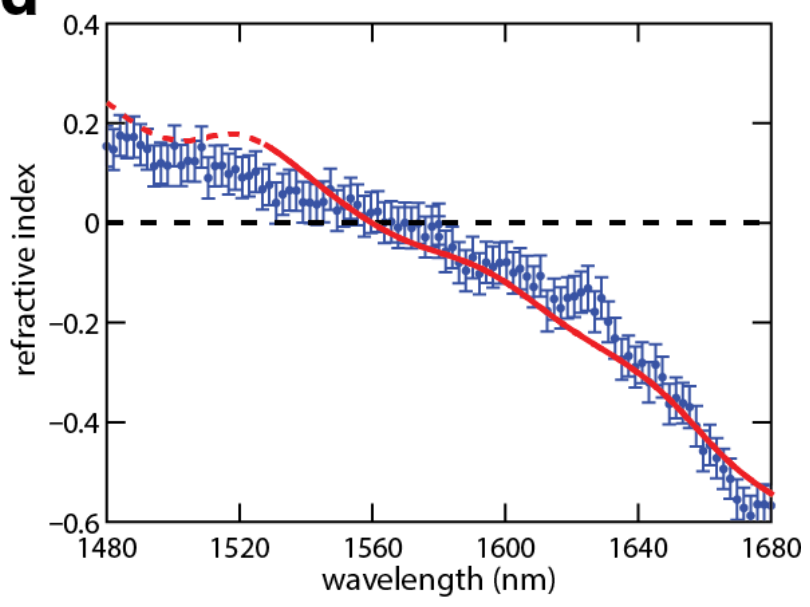

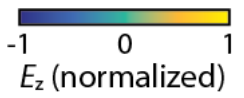

Fig.S24. Change in photonic band structure and extracted $n_{\text {eff }}$ for different pillar radii. (a, c): Band structures of metamaterials with pillar radii $r=210.5 \mathrm{~nm}$ and $190 \mathrm{~nm}$, respectively. The electric monopole mode is indicated by red dots, and the magnetic dipole modes are indicated by blue dots. Insets show $E_{\mathrm{z}}$ profiles corresponding to each band at the $\Gamma$ point. $(\mathbf{b}, \mathbf{d})$ : Effective indices $n_{\text {eff }}$ extracted from refractions through prisms with $r=210.5 \mathrm{~nm}$ and $190 \mathrm{~nm}$, respectively. 
Simulations are shown by red curves, and measured results are shown by blue dots with error bars. The grey bar indicates the bandgap region, as predicted by the band structure.
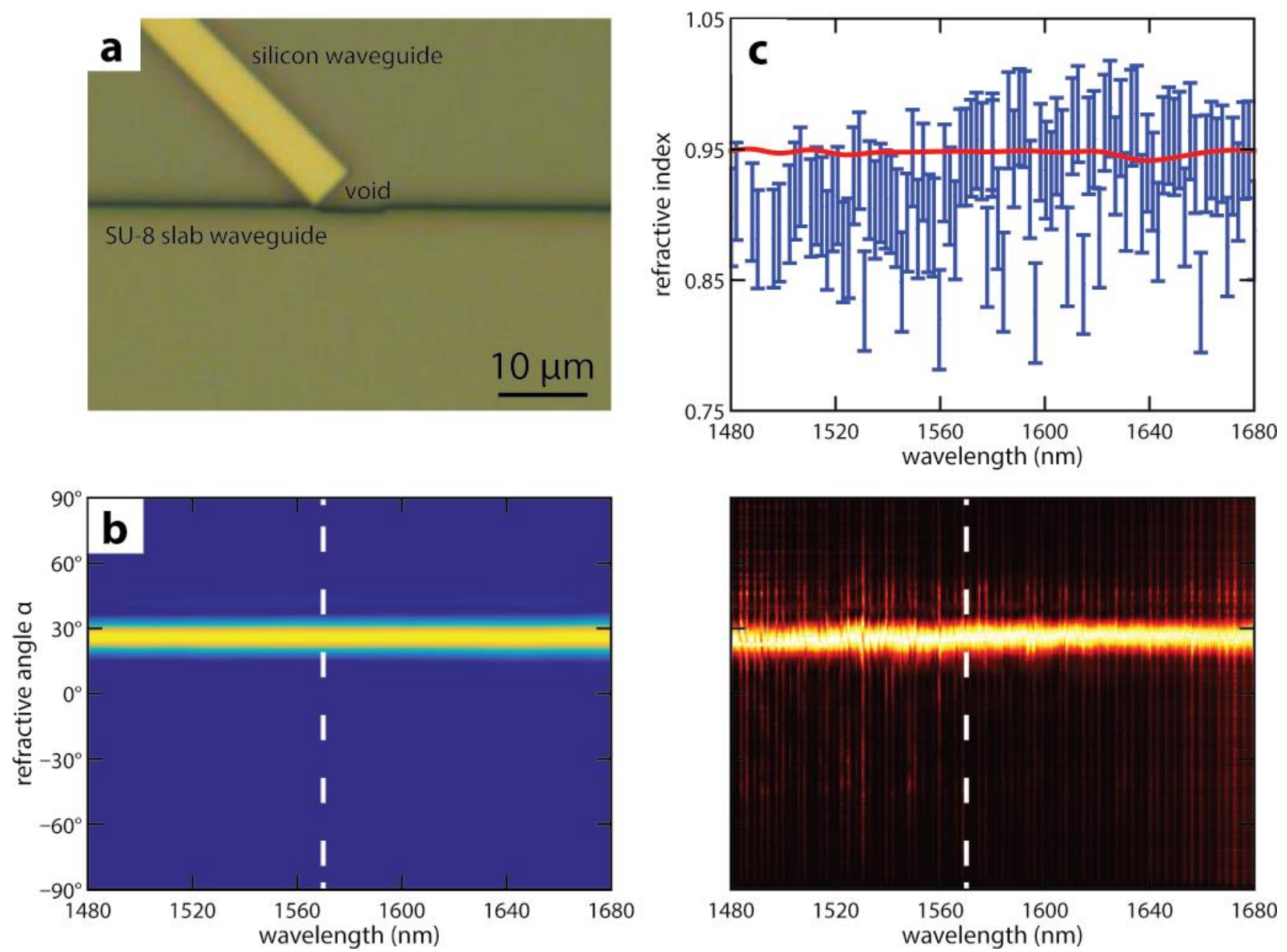

Fig.S25. Optical image, simulation and experimental results of the control experiment. (a) Optical image of the control device. This device is the same as the measurement setup in Fig. 1b, c except for the absence of the metamaterial prism. (b): Simulated (left) and measured (right) far-field patterns. (c) Measured and simulated effective index of the control experiment. The blue dots 
indicate the measured refractive index, with error bars representing the uncertainties in the measurement. The measurement agrees well with the simulated effective index (red line).
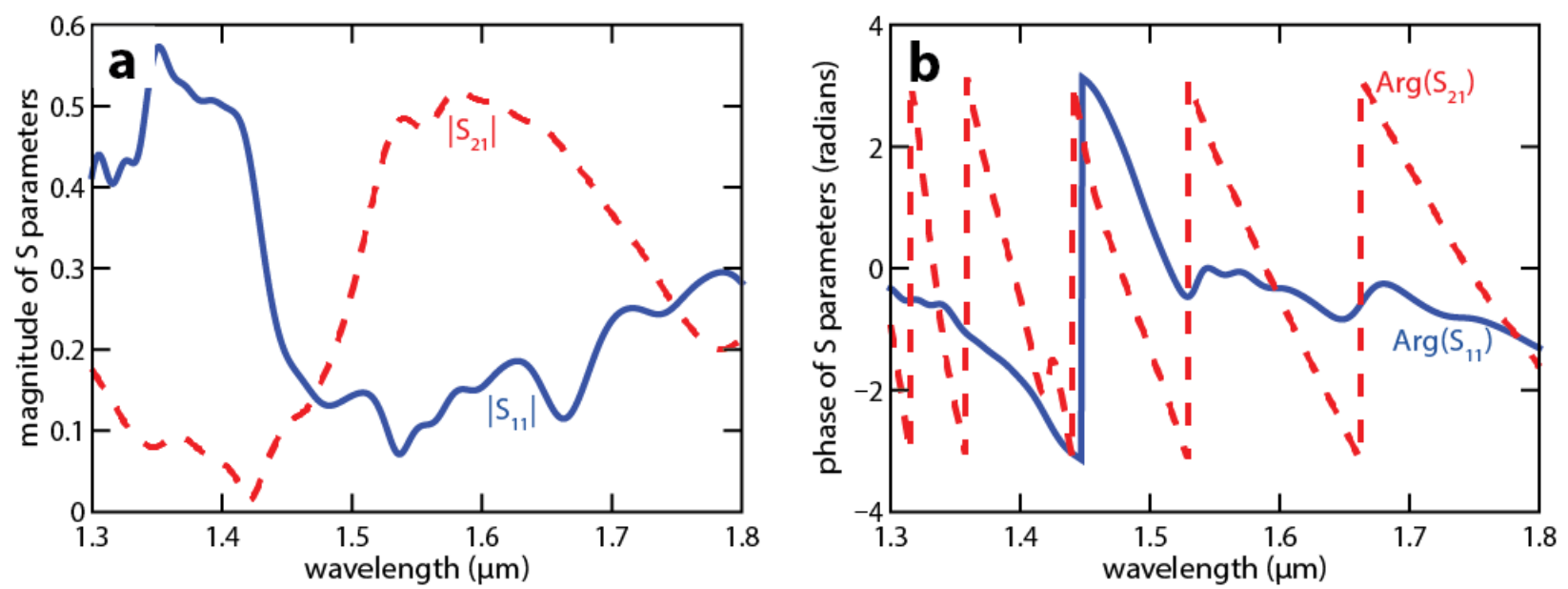

Fig.S26. (a) Magnitude and (b) phase of the numerically calculated reflection $\left(S_{11}\right)$ and transmission $\left(S_{21}\right)$ coefficients ( $S$ parameters) of the presented metamaterial (Fig. 1a). 